\title{
Training Beam Sequence Design for Millimeter-Wave MIMO Systems: A POMDP Framework
}

\author{
Junyeong Seo, Student Member, IEEE, Youngchul Sung ${ }^{\dagger}$, Senior Member, IEEE \\ Gilwon Lee, and Donggun Kim, Student Members, IEEE
}

\begin{abstract}
In this paper, adaptive training beam sequence design for efficient channel estimation in large millimeter-wave (mmWave) multiple-input multiple-output (MIMO) channels is considered. By exploiting the sparsity in large mmWave MIMO channels and imposing a Markovian random walk assumption on the movement of the receiver and reflection clusters, the adaptive training beam sequence design and channel estimation problem is formulated as a partially observable Markov decision process (POMDP) problem that finds non-zero bins in a two-dimensional grid. Under the proposed POMDP framework, optimal and suboptimal adaptive training beam sequence design policies are derived. Furthermore, a very fast suboptimal greedy algorithm is developed based on a newly proposed reduced sufficient statistic to make the computational complexity of the proposed algorithm low to a level for practical implementation. Numerical results are provided to evaluate the performance of the proposed training beam design method. Numerical results show that the proposed training beam sequence design algorithms yield good performance.
\end{abstract}

\section{Keywords}

Millimeter Wave, MIMO, Channel Estimation, Training Signal Design, POMDP

${ }^{\dagger}$ Corresponding author

The authors are with Dept. of Electrical Engineering, KAIST, Daejeon 305-701, South Korea. E-mail:\{jyseo@, ysung@ee., gwlee@, and dg.kim@\}kaist.ac.kr. This research was supported by Basic Science Research Program through the National Research Foundation of Korea (NRF) funded by the Ministry of Education (2013R1A1A2A10060852). 


\section{INTRODUCTION}

The use of the mmWave frequency band allows wide bandwith for high data rates required for future wireless networks. However, mmWave signals experience severe large-scale pathloss compared to lower frequency band signals, which has been a hurdle to using the mmWave band for commercial wireless access networks so far. Recently, active research is going on to use the mmWave band for cellular systems by exploiting advanced hardware and software processing power [1-4]. One of the major techniques to compensate for the large pathloss in the mmWave band is highly directional beamforming based on large antenna arrays [2-4] which can be implemented in small sizes in the mmWave band [5]. Typically such beamforming requires channel state information (CSI) at the transmitter and the receiver, but the CSI is difficult to acquire in the mmWave band due to the propagation directivity and the low signal-to-noise ratio (SNR) before beamforming due to large pathloss. Thus, efficient and accurate channel estimation is one of the key requirements for the success of large mmWave MIMO systems $[2-4]$.

Conventional training-based MIMO channel estimation methods typically assume rich scattering environments or the knowledge* of the channel covariance matrix in the rank-deficient channel case [6-11]. However, such assumption may not be valid in large mmWave MIMO systems due to the high propagation directivity and the narrow beam width associated with large antenna arrays [12]. Typical mmWave channels with large antenna arrays can be modeled as sparse MIMO channels with the virtual channel representation and the directions of ray clusters are unknown to the transmitter and the receiver beforehand [3,4,12,13] (see Fig. 1). Thus, conventionally designed training signals and channel estimation methods aiming at the lower frequency band are less efficient, and the training signal design and channel estimation are more challenging in the mmWave case. One way to identify a sparse channel is to transmit all beam directions sequentially in time and pick the direction of the largest signal magnitude $[14,15]$. However, such a method is not efficient when the number of all possible directions to search is large as in the large mmWave MIMO case. To tackle the challenge of sparse MIMO channel estimation, algorithms based on compressed sensing (CS) theory have recently been developed

${ }^{*}$ The knowledge of the channel covariance matrix in the rank-deficient channel case implies that the propagation ray directions are known to the transmitter a priori. 
$[3,4,12,13]$. In [12], the problem of channel estimation in large mmWave MIMO was formulated to capture the sparse nature of the channel and CS tools were applied to analyze the sparse channel estimation performance. In particular, in [3,4], Alkhateeb et al. proposed a channel estimation and training beam design method for large mmWave MIMO systems based on adaptive CS. In their method, the channel estimation is performed over multiple blocks under the assumption that the channel does not vary over the considered multiple blocks. Each block consists of multiple training beam symbol times so that the sparse recovery is feasible at each block, and the next training beam is adaptively designed based on the previous block observation result by using a space bisection approach which is a reasonable choice to search a propagation ray cluster in the space.

In this paper, exploiting the channel dynamic, we propose a different training beam design and channel estimation paradigm for sparse large mmWave MIMO systems based on a decisiontheoretical framework. We consider a typical time-duplexed training structure, where one slot consists of a block of pilot symbol times and the following symbol times for data transmission [16]. We assume that still a highly directional narrow pilot beam should be transmitted at each training symbol time to compensate for large pathloss and obtain a reasonable quality channel gain estimate in the mmWave band. Then, the training beam design problem reduces to the problem of choosing the directions of the pilot beams in the space to find the actual propagation paths generated by line-of-sight and/or reflection clusters, as shown in Fig. 1. Specifically, with the virtual channel representation [12], the sparse channel estimation reduces to finding the locations and values of the non-zero valued bins in a two-dimensional grid. Our main idea is to impose a Markovian random walk structure on the movement of the mobile station and the reflection clusters ${ }^{\dagger}$. In the proposed scheme, a set of pilot beams is transmitted at each slot and the set of pilot beams at the current slot is adaptively and optimally determined based on the observation over all the previous slots to maximize a properly defined reward accumulated over a given communication period. Since we cannot observe all possible ray directions in the space at each slot and the identification of a path can be wrong, the pilot beam design under this formulation reduces to a partially observable Markov decision process (POMDP) [17], and the

\footnotetext{
${ }^{\dagger}$ This assumption seems reasonable when we consider the physics of the mobile station or the reflection clusters. For the example of a pedestrian user with a certain speed of walking, the propagation ray directions at the next slot changes from the current directions and this uncertain change can be captured as a random walk.
} 
theory of POMDP can be applied to the pilot beam design problem for large mmWave MIMO. Under the proposed POMDP formulation optimal and suboptimal greedy strategies for training beam sequence design for sparse large MIMO channel estimation are derived. However, the direct application of standard POMDP theory yields an intractable number of states and unfeasible complexity. Thus, exploiting the specific structure of the mobile communication channel and deriving a new reduced-size sufficient statistic for the decision process, we develop a greedy algorithm with significantly reduced complexity so that the proposed algorithm can practically be operated. Numerical results show that the proposed low complexity suboptimal algorithm yields comparable performance relative to the optimal algorithm, and the proposed training beam design algorithms efficiently estimate and tract sparse MIMO channels. (A preliminary version of this work was submitted to ICC 2015 [18].)

Notations and Organization We will use standard notational conventions in this paper. Vectors and matrices are written in boldface with matrices in capitals. For a matrix $\mathbf{A}, \mathbf{A}^{T}, \mathbf{A}^{H}$, $[\mathbf{A}]_{i j}, \mathbf{A}(:, k)$, and $\operatorname{tr}(\mathbf{A})$ indicate the transpose, conjugate transpose, the element of the $i$-th row and the $j$-th column, the $k$-th column, and trace of $\mathbf{A}$, respectively. $\mathbf{I}_{n}$ stands for the identity matrix of size $n$ and $\mathbf{1}_{n}$ stands for the matrix of size $n$ whose entries are all one. $\mathbf{A} \otimes \mathbf{B}$ is the Kronecker product of $\mathrm{A}$ and $\mathrm{B}$. The notation $\mathrm{x} \sim \mathcal{C}(\boldsymbol{\mu}, \boldsymbol{\Sigma})$ means that $\mathrm{x}$ is complex Gaussian distributed with mean vector $\boldsymbol{\mu}$ and covariance matrix $\boldsymbol{\Sigma}$. $\mathbb{E}\{\cdot\}$ denotes the expectation. $|\mathbf{a}|$ and $\|\mathbf{a}\|_{0}$ denote the number of elements and the number of non-zero elements of a, respectively. $\iota:=\sqrt{-1}$.

This paper is organized as follows. In Section II, the system model is explained. In Section III, the optimal training beam sequence design problem is formulated as a POMDP problem. In Section IV, optimal and suboptimal strategies are presented and a greedy pilot beam design algorithm with low complexity is derived. Numerical results are provided in Section V, followed by conclusions in Section VI.

\section{System Model}

\section{A. Sparse Channel Modeling in Large mmWave MIMO Systems}

We consider a mmWave MIMO system, where a transmitter equipped with a uniform linear array (ULA) of $N_{t}$ antennas communicates to a receiver equipped with a ULA of $N_{r}$ antennas. 
The received signal at symbol time $n$ is then given by

$$
\mathbf{y}_{n}=\mathbf{H}_{n} \mathbf{x}_{n}+\mathbf{n}_{n}, \quad n=1,2, \cdots,
$$

where $\mathbf{H}_{n}$ is the $N_{r} \times N_{t}$ MIMO channel matrix at time $n, \mathbf{x}_{n}$ is the $N_{t} \times 1$ transmit symbol vector at time $n$ with a power constraint $\operatorname{tr}\left(\mathbb{E}\left\{\mathbf{x}_{n} \mathbf{x}_{n}^{H}\right\}\right) \leq P_{t}$, and $\mathbf{n}_{n}$ is the $N_{r} \times 1$ Gaussian noise vector at time $n$ from $\mathcal{C N}\left(\mathbf{0}, \sigma_{N}^{2} \mathbf{I}_{N_{r}}\right)$.

A physical multipath channel accurately modeling $\mathbf{H}_{n}$ is given by [19,20]

$$
\mathbf{H}_{n}=\sqrt{N_{t} N_{r}} \sum_{l=1}^{L} \alpha_{n, l} \mathbf{a}_{R X}\left(\theta_{n, l}^{r}\right) \mathbf{a}_{T X}^{H}\left(\theta_{n, l}^{t}\right),
$$

where $\alpha_{n, l} \sim \mathcal{C N}\left(0, \xi^{2}\right)$ is the complex gain of the $l$-th path at time $n$, and $\theta_{n, l}^{r}$ and $\theta_{n, l}^{t}$ are the angle-of-arrival (AoA) and angle-of-departure (AoD) normalized directions of the $l$-th path at time $n$ for the receiver and the transmitter, respectively. Here, the normalized direction $\theta$ is related to the physical angle $\phi \in[-\pi / 2, \pi / 2]$ as

$$
\theta=\frac{d \sin (\phi)}{\lambda}
$$

where $d$ and $\lambda$ are the spacing between two adjacent antenna elements and the signal wavelength, respectively. We assume $\frac{d}{\lambda}=\frac{1}{2}$ and thus, the range of $\theta$ is $\left[-\frac{1}{2}, \frac{1}{2}\right]$. In (2), $\mathbf{a}_{R X}\left(\theta^{r}\right)$ and $\mathbf{a}_{T X}\left(\theta^{t}\right)$ are the receiver response and the transmitter steering vector in normalized directions $\theta^{r}$ and $\theta^{t}$, respectively, which are defined as [20]

$$
\begin{aligned}
& \mathbf{a}_{R X}\left(\theta^{r}\right)=\frac{1}{\sqrt{N_{r}}}\left[1, e^{-\iota 2 \pi \theta^{r}}, \cdots, e^{-\iota\left(N_{r}-1\right) 2 \pi \theta^{r}}\right]^{T}, \\
& \mathbf{a}_{T X}\left(\theta^{t}\right)=\frac{1}{\sqrt{N_{t}}}\left[1, e^{-\iota 2 \pi \theta^{t}}, \cdots, e^{-\iota\left(N_{t}-1\right) 2 \pi \theta^{t}}\right]^{T} .
\end{aligned}
$$

Note that $\left\|\mathbf{a}_{R X}\left(\theta^{r}\right)\right\|=\left\|\mathbf{a}_{T X}\left(\theta^{t}\right)\right\|=1$. Neglecting the angle quantization error, we can map the physical MIMO channel matrix $\mathbf{H}_{n}$ to a virtual channel matrix (VCM) $\widetilde{\mathbf{H}}_{n}$ through the following relationship [19]

$$
\mathbf{H}_{n}=\mathbf{A}_{R} \widetilde{\mathbf{H}}_{n} \mathbf{A}_{T}^{H}
$$

where $\mathbf{A}_{R}=\left[\mathbf{a}_{R X}\left(\tilde{\theta}_{1}^{r}\right), \cdots, \mathbf{a}_{R X}\left(\tilde{\theta}_{N_{r}}^{r}\right)\right], \tilde{\theta}_{i}^{r}=-\frac{1}{2}+\frac{i-1}{N_{r}}$ for $i=1, \cdots, N_{r}$, and $\mathbf{A}_{T}=$ $\left[\mathbf{a}_{T X}\left(\tilde{\theta}_{1}^{t}\right), \cdots, \mathbf{a}_{T X}\left(\tilde{\theta}_{N_{t}}^{t}\right)\right], \tilde{\theta}_{j}^{t}=-\frac{1}{2}+\frac{j-1}{N_{t}}$ for $j=1, \cdots, N_{t}$. (From here on, we will neglect the angle quantization error.) The $(i, j)$-th element of $\widetilde{\mathbf{H}}_{n}$ represents the channel gain scaled by 


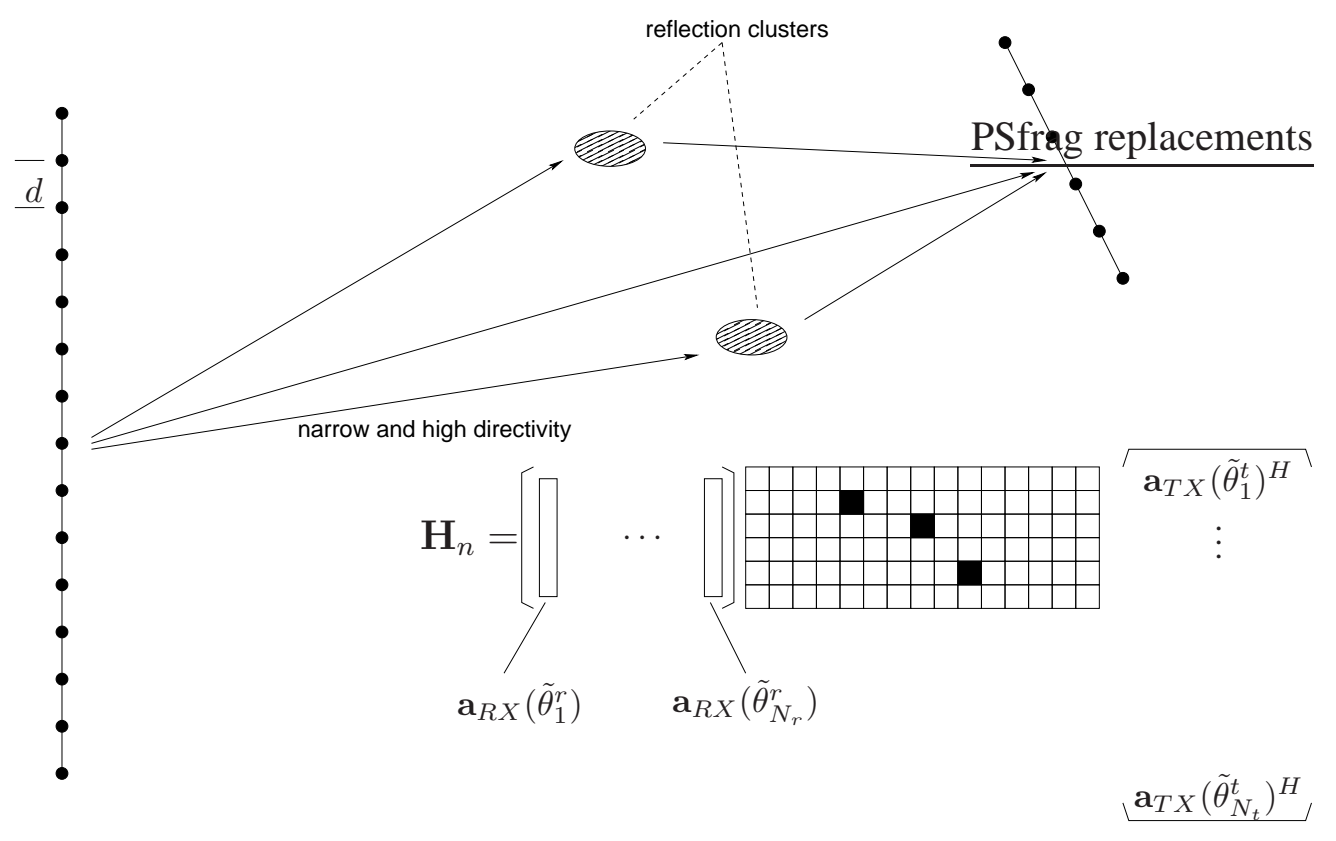

Fig. 1

THE CONSIDERED MMWAVE CHANNEL MODEL

$\sqrt{N_{t} N_{r}}$ when the virtual angles seen by the receiver and the transmitter are $\tilde{\theta}_{i}^{r}$ and $\tilde{\theta}_{j}^{t}$, respectively. For the element of $\widetilde{\mathbf{H}}_{n}$ corresponding to the $l$-th propagation path, the element value is given by $\sqrt{N_{t} N_{r}} \alpha_{n, l}$. The physical channel model (2) induces a sparse property to the virtual channel representation, given by

$$
\sum_{j=1}^{N_{t}}\left\|\widetilde{\mathbf{H}}_{n}(:, j)\right\|_{0}=L .
$$

That is, there are only $L\left(\ll N_{t} N_{r}\right)$ non-zero elements among the $N_{t} N_{r}$ entries of the VCM $\widetilde{\mathbf{H}}_{n}$. Therefore, for large $N_{t}$ and $N_{r}$, it is generally difficult to find the locations of the $L$ non-zero elements in $\widetilde{\mathbf{H}}_{n}$ without an exhaustive search.

For the sake of simplicity, with the cellular downlink in mind, we assume that $N_{r} \ll N_{t}$ and the receiver has $N_{r}$ monolithic microwave integrated circuit (MMIC) RF chains so that the receiver can implement the filter bank $\mathbf{A}_{R}^{H}$. Then, the receiver-filtered signal at the filter bank output is given by

$$
\mathbf{y}_{n}^{\prime}:=\mathbf{A}_{R}^{H} \mathbf{y}_{n}=\widetilde{\mathbf{H}}_{n} \mathbf{A}_{T}^{H} \mathbf{x}_{n}+\mathbf{n}_{n}^{\prime}
$$

where $\mathbf{n}_{n}^{\prime}=\mathbf{A}_{R}^{H} \mathbf{n}_{n}$. 
Now, since the receiver checks all the possible (quantized) ray directions, the remaining problem is to design the transmit training beam sequence $\left\{\mathbf{x}_{n} \mid n \in T_{\mathcal{P}}\right\}$ for estimating the sparse channel, where $T_{\mathcal{P}}$ is the set of symbol times allocated to training signal transmission.

\section{B. The Considered Dynamic Channel Model}

To design an efficient training beam sequence for estimating the sparse channel presented in Section II-A, we exploit the channel dynamic and model the channel dynamic by imposing a block Markovian structure on the VCM $\widetilde{\mathbf{H}}_{n}$. That is, $\widetilde{\mathbf{H}}_{n}$ is constant over one slot consisting of $M_{s}$ symbols. Let us denote the VCM at slot $k$ by $\widetilde{\mathbf{H}}_{(k)}$. The VCM $\widetilde{\mathbf{H}}_{(k)}$ at slot $k$ changes to $\widetilde{\mathbf{H}}_{(k+1)}$ at slot $k+1$ in a Markovian manner. Here, the conventional block Gauss-Markov process or state-space channel model widely used in time-varying channel estimation $[9,10]$ is inappropriate to model the sparse mmWave MIMO channel, and the sparsity of the mmWave MIMO channel should be captured in the Markov model. Focusing on the dynamic of the locations of the non-zero elements of the VCM rather than the values ${ }^{\ddagger}$ and considering that the non-zero elements in $\widetilde{\mathbf{H}}_{(k)}$ are associated with the line-of-sight (LOS) and reflection clusters, we assume the following model:

Assumption 1: Each of the $L$ paths (or the locations of the non-zero elements) in $\widetilde{\mathbf{H}}_{(k)}$ moves from the current column location to another column location in $\widetilde{\mathbf{H}}_{(k+1)}$ in a Markovian manner with a transition probability, and the transition probability does not change over the considered period of time of total $T$ slots. Furthermore, the transition of each path is independent.

Here, we do not consider the row-wise transition of the non-zero bins in the VCM because we assume that the receiver has a filter bank that checks all AoA directions in parallel at each symbol time. The rationale for the above assumption is that each propagation path is generated by either the LOS or a reflection cluster and the physical movement of the LOS or a reflection cluster can be modelled as a random walk in space. This random walk translates into each nonzero bin's random walk in the VCM. Thus, in the proposed model, the column location of the $l$-th path follows a random walk on $\left\{1,2, \cdots, N_{t}\right\}$ and the path gain of the $l$-th path is given by the sequence $\left\{\alpha_{(1), l}, \alpha_{(2), l}, \cdots, \alpha_{(k), l}, \cdots\right\}$, where $\alpha_{(k), l}$ is the complex gain of the $l$-th path at slot $k$.

\footnotetext{
${ }^{\ddagger}$ The value will be obtained with reasonable quality once the correct direction is hit by the pilot beam with high power.
} 


\section{B.1 The Case of a Single Path}

First, consider the single path case, i.e., $L=1$. In this case, the number $N$ of states is $N_{t}$ because we have $N_{t}$ columns in the VCM. Thus, the set $\mathcal{S}$ of all possible states is given by

$$
\mathcal{S}=\left\{1,2, \cdots, N_{t}\right\}
$$

where state $i$ denotes the state that the path is located in the $i$-th column of the VCM. With the set $\mathcal{S}$ of states defined, the $(i, j)$-th element of the $N \times N$ state transition probability matrix $\mathbf{P}$ is defined as

$$
p_{i j}=\operatorname{Pr}\left\{S_{k+1}=j \mid S_{k}=i\right\}, \quad i, j \in \mathcal{S},
$$

where $S_{k}$ and $S_{k+1}$ denote the states of slots $k$ and $k+1$, respectively. The transition probability matrix $\mathbf{P}$ captures the characteristics of the movement behavior of the path and hence it should be designed carefully by considering the physics of the receiver and reflection cluster movement. For example, vehicular channels or pedestrian channels with certain speeds will have different the values in $\mathbf{P}$. Intuitively, it is reasonable to design $\mathbf{P}$ so that the transition probability from the $i$-th column to the $j$-th column monotonically decreases as $|i-j|$ increases. That is, it is more likely to shift to a nearby column with continuous movement. Ignoring the possibility of the path's movement with a large AoD change per slot, we can model the transition probability matrix as a banded matrix. In this case, for example, we can consider the transition probability matrix with exponential decay given by

$$
\mathbf{P}_{\beta}^{B}=\left[\begin{array}{cccccccccccc}
0 & \alpha \beta^{B} & \ldots & \alpha \beta & \alpha & \alpha \beta & \cdots & \alpha \beta^{B} & 0 & \cdots & \\
\cdots & 0 & \alpha \beta^{B} & \cdots & \alpha \beta & \alpha & \alpha \beta & \cdots & \alpha \beta^{B} & 0 & \cdots \\
& \cdots & 0 & \alpha \beta^{B} & \cdots & \alpha \beta & \alpha & \alpha \beta & \cdots & \alpha \beta^{B} & 0 \\
& & & & & & & \ddots & & &
\end{array}\right],
$$

where $B$ is the bandwidth of the matrix and $\beta \in[0,1)$ is the exponential decaying factor. (The value of $\alpha$ and the corner of $\mathbf{P}_{\beta}^{B}$ should be obtained properly so that the sum of each row is one.) When we want to model the random path appearance from an arbitrary direction, we can use

$$
\mathbf{P}_{\beta, \lambda}^{B}=(1-\lambda) \mathbf{P}_{\beta}^{B}+\lambda \frac{1}{N_{t}} \mathbf{1}_{N_{t}}
$$




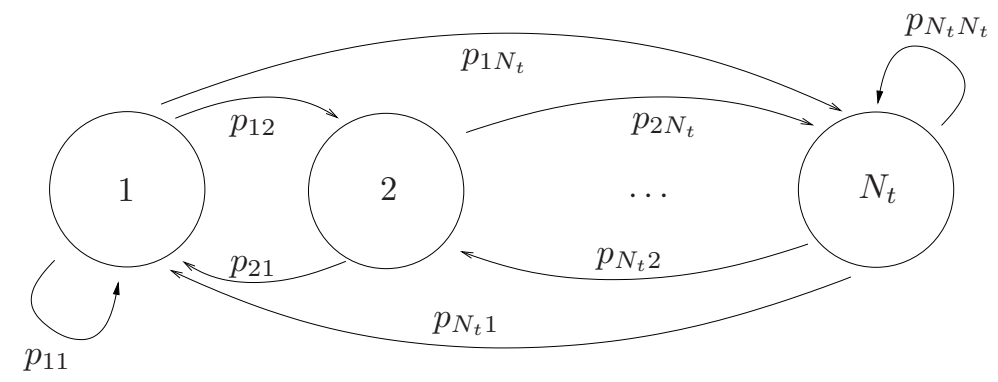

Fig. 2

The Markov Chain Model For the Channel Dynamic: The single Path CASE

where $\mathbf{1}_{N_{t}}$ is the $N_{t} \times N_{t}$ matrix whose entries are all one and $\lambda \in[0,1]$. Note that the proposed model can capture static channels by setting $\mathbf{P}=\mathbf{I}$. Fig. 2 shows the Markov chain in the single path case.

\section{B.2 The Case of Multiple Paths}

Now consider the multi-path case, i.e., $L \geq 2$. We allow multiple paths to merge on and diverge from a column of the VCM. In this case, the set $\mathcal{S}$ of all possible states is given by

$$
\mathcal{S}=\left\{\left(i_{1}, i_{2}, \cdots, i_{L}\right), i_{1}, i_{2}, \cdots, i_{L}=1,2, \cdots, N_{t}\right\}
$$

where state $\left(i_{1}, \cdots, i_{L}\right)$ denotes that the $l$-th path is located at the $i_{l}$-th column of the VCM for $l=1, \cdots, L$, and the cardinality $N$ of $\mathcal{S}$ is $N_{t}^{L}$. Under the assumption of independent path movement the state transition probability in the $L$-path case is given by

$$
\begin{aligned}
& \operatorname{Pr}\left\{S_{k+1}=\left(j_{1}, \cdots, j_{L}\right) \mid S_{k}=\left(i_{1}, \cdots, i_{L}\right)\right\} \\
& \quad=p_{i_{1} j_{1}} p_{i_{2} j_{2}} \times \cdots \times p_{i_{L} j_{L}}
\end{aligned}
$$

where $p_{i j}$ denotes the transition probability that a path moves from the $i$-th column to the $j$-th column of the VCM at the next slot, defined in (9). States $\left(i_{1}, \cdots, i_{L}\right), i_{1}, \cdots, i_{L}=1, \cdots, N_{t}$, can be enumerated as states $\mathbf{s}^{(i)}, i=1,2, \cdots, N=N_{t}^{L}$. Thus, $\mathcal{S}$ can also be expressed for notational simplicity as

$$
\mathcal{S}=\left\{\mathbf{s}^{(1)}, \mathbf{s}^{(2)}, \cdots, \mathbf{s}^{(N)}\right\}
$$

Fig. 3 shows an example of the transition of each path when $L=3$ and $N_{t}=N_{r}=7$. In this example, the AoD directions of the 1 st, 2 nd, and 3 rd path move from $\tilde{\theta}_{5}^{t}$ to $\tilde{\theta}_{6}^{t}$, from $\tilde{\theta}_{3}^{t}$ to 

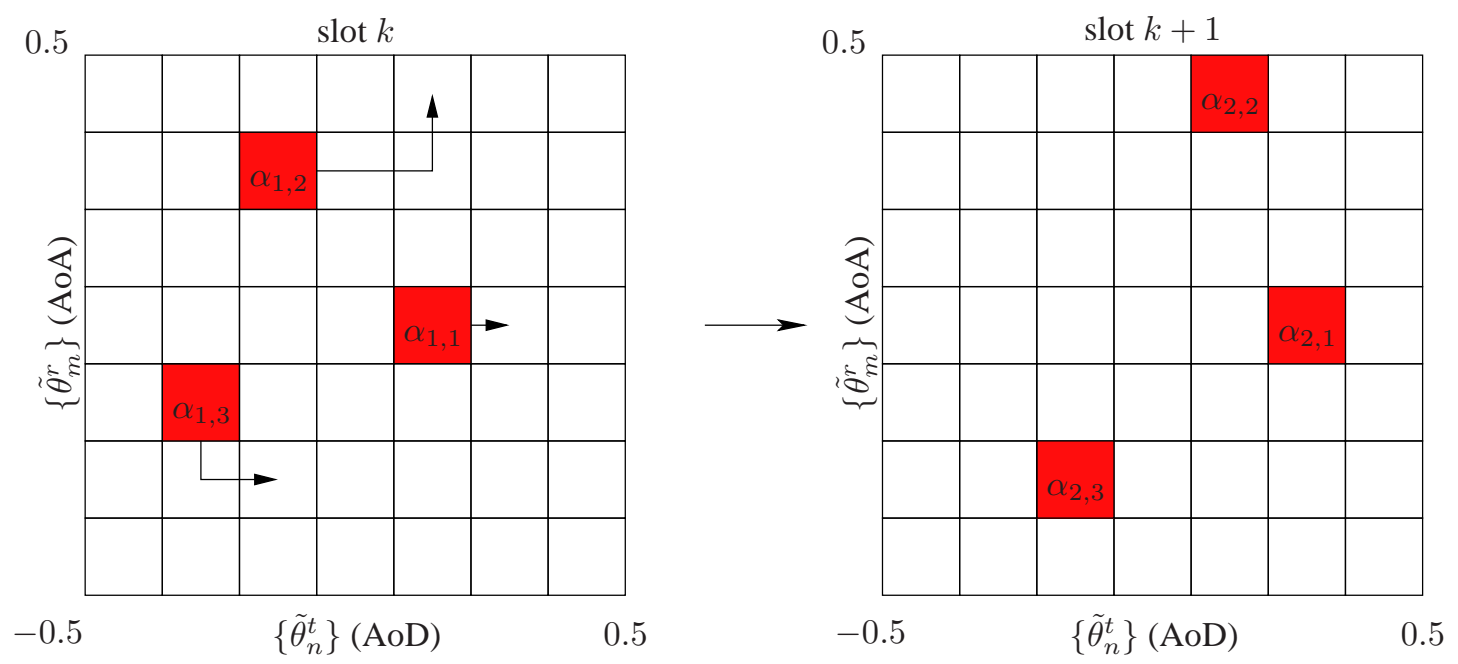

Fig. 3

An illustration of THE tRANSition of EACH PATH $\left(L=3\right.$ AND $\left.N_{t}=N_{r}=7\right)$

$\tilde{\theta}_{5}^{t}$, and from $\tilde{\theta}_{2}^{t}$ to $\tilde{\theta}_{3}^{t}$, respectively. The probability of this transition is $p_{56} \times p_{35} \times p_{23}$ under the assumption of independent movement of each path.

\section{Channel Sensing with Pilot Beams}

We here explain the transmission structure. We consider a typical time-duplexed training structure, where one slot of size $M_{s}$ slots consists of a block of $M_{p}\left(L \leq M_{p} \ll N_{t}\right)$ pilot symbol times and the remaining symbol times of the slot are used for data transmission [16]. We assume that the transmitter picks one column of $\mathbf{A}_{T}$ as the pilot beam at each pilot symbol time and search one column of $\widetilde{\mathbf{H}}_{(k)}$ at each pilot symbol time, i.e., $\mathbf{x}_{n} \in\left\{\sqrt{P_{t}} \mathbf{a}_{T X}\left(\tilde{\theta}_{1}^{t}\right), \cdots\right.$, $\left.\sqrt{P_{t}} \mathbf{a}_{T X}\left(\tilde{\theta}_{N_{t}}^{t}\right)\right\}$ for the training time. Hence, $M_{p}$ columns of $\mathbf{A}_{T}$ are selected as the $M_{p}$ pilot beams in a slot. The reason for this assumption is that we assume that the pathloss is severely large in the mmWave band and thus the pilot beam as well as the data-transmitting beam should be highly directional to compensate for the large pathloss and obtain a channel gain estimate of reasonable quality, unless $P_{t}$ is extremely high. (The relaxation of this assumption will be discussed in Section IV-C.) When $\sqrt{P_{t}} \mathbf{a}_{T X}\left(\tilde{\theta}_{i_{m}}^{t}\right)$ is the pilot beam at the $m$-th symbol time of slot $k$, from (8), the receiver filter-bank output is given by

$$
\mathbf{y}_{(k)}^{\prime}[m]=\sqrt{P_{t}} \widetilde{\mathbf{H}}_{(k)}\left(:, i_{m}\right)+\mathbf{n}_{(k)}^{\prime}[m]
$$




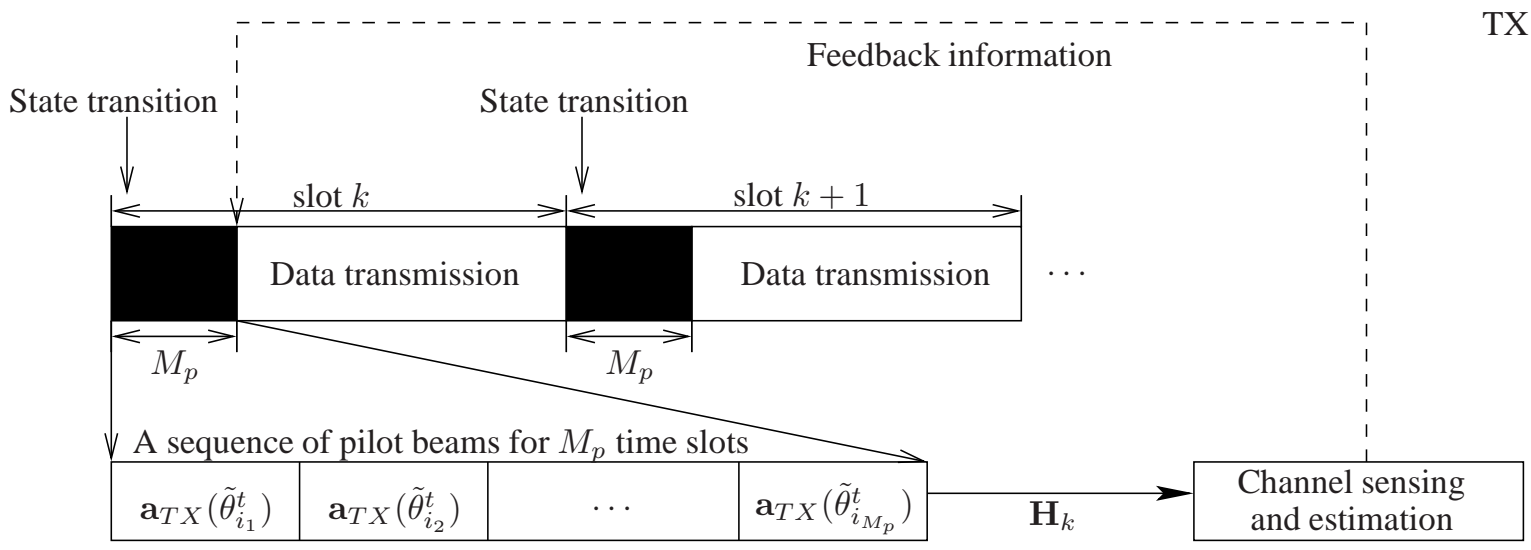

Fig. 4

THE OVERALL PROCESS OF TRAINING AND DATA TRANSMISSION

where $\mathbf{y}_{(k)}^{\prime}[m]$ and $\mathbf{n}_{(k)}^{\prime}[m]$ denote the signal and the noise at the receiver filter-bank output at the $m$-th pilot symbol time of slot $k$, and $\widetilde{\mathbf{H}}_{(k)}\left(:, i_{m}\right)$ is the $i_{m}$-th column of $\widetilde{\mathbf{H}}_{(k)}$.

During the training period, the receiver senses and estimates the $M_{p}$ columns of the VCM corresponding to the $M_{p}$ pilot beams. Then, the receiver feedbacks the sensing results and estimated channel gains to the transmitter. Finally, the transmitter sends data and adapts the pilot beams for the next slot based on the information from the receiver. At the next slot, the process is repeated. The whole process of training and data transmission is depicted in Fig. 4.

Now, the problem is how to optimally design the sequence of pilot beams for $M_{p}$ symbol times

for each slot. Since $M_{p} \ll N_{t}$, we can only sense a few columns of $\widetilde{\mathbf{H}}_{(k)}$ at slot $k$. Hence, $M_{p}$ pilot beams for each slot should be designed judiciously by exploiting the channel dynamic and the available information in all the previous slots. In the next section, we propose an analytical framework for optimal pilot beam sequence design based on POMDP theory.

\section{POMdP Formulation for Training BeAm SEQuence Design}

In this section, we formulate a relevant POMDP problem for the training beam sequence design problem for estimation of large sparse mmWave MIMO channels.

\section{A. The Action Space and the Observation Space}

In the previous section, we assumed that $M_{p}$ columns of $\mathbf{A}_{T}$ are selected as the pilot beam sequence for the $M_{p}$ pilot symbol times for each slot. This is equivalent to selecting $M_{p}$ columns 
of the VCM to be sensed by the training beam sequence at each slot. Let us denote the selected column indices of the VCM by

$$
\mathbf{a}=\left[a_{1}, a_{2}, \cdots, a_{M_{p}}\right]
$$

where $a_{m}$ is the index of the column of the VCM that is sensed at the $m$-th pilot symbol time. The vector a represents the action that we perform at each slot and is referred to as the action vector. Note that there exist $\left(\begin{array}{l}N_{t} \\ M_{p}\end{array}\right)$ possible a's and the optimal training beam sequence design problem reduces to choosing the best a for each slot under the considered optimality criterion.

After the training period of each slot is finished, the receiver returns feedback information to the transmitter. The feedback information contains the detection result about the existence of paths in the selected columns of the VCM and the complex gains of the detected paths. Then, the transmitter uses the channel gain information of the detected paths for transmit beamforming during the data transmission period and uses the feedback information about the existence of paths to select the training beam indices for the next slot in an adaptive manner. The second feedback information can be modeled as

$$
\mathbf{o}=\left[o_{1}, o_{2}, \cdots, o_{M_{p}}\right] \in\{0,1\}^{M_{p}},
$$

where $o_{m}=1$ indicates that a path is detected by the training beam transmitted at the $m$-th pilot symbol time, and otherwise $o_{m}=0$. Since we have $2^{M_{p}}$ possible vectors for $\mathbf{o}$, the observation space is given by

$$
\mathcal{O}=\left[\mathbf{o}^{(1)}, \mathbf{o}^{(2)}, \cdots, \mathbf{o}^{\left(2^{M_{p}}\right)}\right]
$$

When the state of the VCM is $\mathbf{s}^{(i)}$ and the action vector a is used for the pilot beam sequence for the slot, the probability that the transmitter observes the feedback information $\mathbf{o}^{(j)}$ is denoted as $q_{i j}^{\text {a }}$, i.e.,

$$
q_{i j}^{\mathbf{a}} \triangleq \operatorname{Pr}\left\{\mathbf{o}=\mathbf{o}^{(j)} \mid \mathbf{s}^{(i)}, \mathbf{a}\right\} \quad \text { for } \mathbf{s}^{(i)} \in \mathcal{S}, \mathbf{o}^{(j)} \in \mathcal{O}
$$

This probability depends on the detector used to identify the existence of a path at the receiver, and will be discussed next. 


\section{A.1 The Channel Sensor at the Receiver}

When the action vector $\mathbf{a}=\left[a_{1}, \cdots, a_{M_{p}}\right]$ is used for the current slot, the receiver filter-bank output at the $m$-th pilot symbol time of the current slot is given from (15) by

$$
\mathbf{y}_{(k)}^{\prime}[m]=\sqrt{P_{t}} \widetilde{\mathbf{H}}_{(k)}\left(:, a_{m}\right)+\mathbf{n}_{(k)}^{\prime}[m]
$$

where $\mathbf{n}_{(k)}^{\prime}[m] \sim \mathcal{C N}\left(0, \sigma_{N}^{2} \mathbf{I}\right)$ since $\mathbf{A}_{R}$ is a unitary matrix. Based on $\mathbf{y}_{(k)}^{\prime}[m]$ the receiver tests the existence of a path at each AoA by checking each element of the $N_{r} \times 1$ vector $\mathbf{y}_{(k)}^{\prime}[\mathrm{m}]$. Then, the detection problem for each element of $\mathbf{y}_{(k)}^{\prime}[m]$ is given by

$$
\begin{cases}\mathcal{H}_{0}: & p\left(\mathbf{y}_{(k)}^{\prime}[m](n) \mid \text { empty }\right) \sim \mathcal{C N}\left(0, \sigma_{N}^{2}\right), \\ \mathcal{H}_{1}: & p\left(\mathbf{y}_{(k)}^{\prime}[m](n) \mid \text { non-empty }\right) \sim \mathcal{C N}\left(0, P_{t} N_{t} N_{r} \xi^{2}+\sigma_{N}^{2}\right)\end{cases}
$$

where $\mathbf{y}_{(k)}^{\prime}[m](n)=y_{R}(n)+\iota y_{I}(n)$ is the $n$-th element of $\mathbf{y}_{(k)}^{\prime}[m], n=1,2, \cdots, N_{r}$. We assume that a Neyman-Pearson detector with size (i.e., false alarm probability) $P_{F A}[21,22]$ is adopted to test (19). Then, the detector is given by [21]

$$
\delta_{N P}= \begin{cases}1, & \left|\mathbf{y}_{(k)}^{\prime}[m](n)\right|^{2} \geq \tau \\ 0, & \text { otherwise }\end{cases}
$$

where $\tau=\sigma_{N}^{2} \Gamma^{-1}\left(1 ; 1-P_{F A}\right)$ since $\left|\mathbf{y}_{(k)}^{\prime}[m](n)\right|^{2}=y_{R}^{2}(n)+y_{I}^{2}(n) \sim \operatorname{gamma}\left(1,1 / \sigma_{N}^{2}\right)$ under $\mathcal{H}_{0}$. Here, $\Gamma^{-1}$ is the inverse function of the incomplete gamma function $\Gamma(x ; t)$. The corresponding miss detection probability is given by [21]

$$
P_{M D}=\Gamma\left[1 ; \frac{1}{1+P_{t} N_{t} N_{r} \xi^{2} / \sigma_{N}^{2}} \Gamma^{-1}\left(1 ; 1-P_{F A}\right)\right],
$$

where $P_{t} N_{t} N_{r} \xi^{2} / \sigma_{N}^{2}$ is the path SNR incorporating the transmit power, path gain, transmit beamforming and receive beamforming.

Now consider $q_{i j}^{\mathbf{a}}$ in (17).

$$
\begin{aligned}
q_{i j}^{\mathbf{a}} & =\operatorname{Pr}\left\{\mathbf{o}=\mathbf{o}^{(j)} \mid \mathbf{s}^{(i)}, \mathbf{a}\right\}, \\
& =\operatorname{Pr}\left\{o_{m}=o_{m}^{(j)}, m=1, \cdots, M_{p} \mid \mathbf{s}^{(i)}, \mathbf{a}\right\}, \\
& \stackrel{(a)}{=} \operatorname{Pr}\left\{o_{1}=o_{1}^{(j)} \mid \mathbf{s}^{(i)}, \mathbf{a}\right\} \times \cdots \times \operatorname{Pr}\left\{o_{M_{p}}=o_{M_{p}}^{(j)} \mid \mathbf{s}^{(i)}, \mathbf{a}\right\}, \\
& =\operatorname{Pr}\left\{o_{1}=o_{1}^{(j)} \mid \mathbf{s}^{(i)}, a_{1}\right\} \times \cdots \times \operatorname{Pr}\left\{o_{M_{p}}=o_{M_{p}}^{(j)} \mid \mathbf{s}^{(i)}, a_{M_{p}}\right\},
\end{aligned}
$$




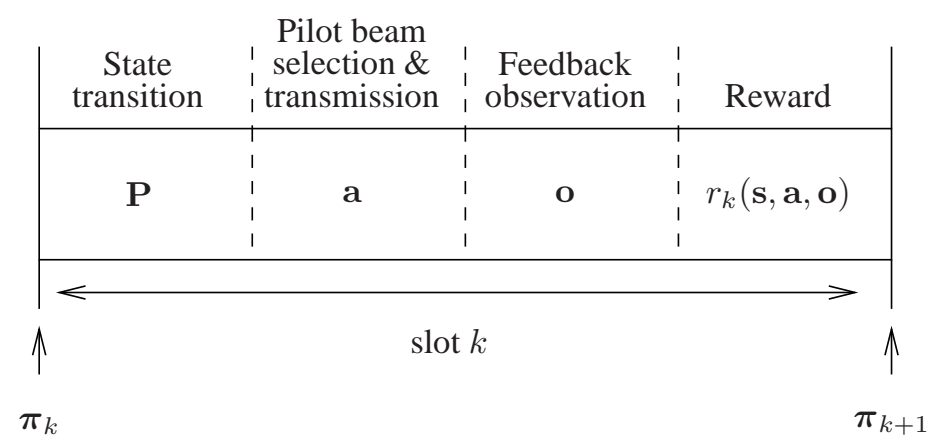

Fig. 5

THE SEQUENCE OF OPERATION AT BLOCK $k$

where step (a) follows since only the noise randomness remains once the state is given, and the receiver noise is assumed to be independent over the $M_{p}$ pilot symbol times. $\operatorname{Pr}\left\{o_{m}=\right.$ $\left.o_{m}^{(j)} \mid \mathbf{s}^{(i)}, a_{m}\right\}$ is computed as follows.

$$
\begin{aligned}
\operatorname{Pr}\left\{o_{m}=0 \mid \mathbf{s}^{(i)}, a_{m}\right\} & =\operatorname{Pr}\left\{\delta_{N P}=0 \mid \mathcal{H}_{0}\right\}^{N_{r}-N_{\mathbf{s}^{(i)}, a_{m}}^{B I N} \operatorname{Pr}} \operatorname{Pr}\left\{\delta_{N P}=0 \mid \mathcal{H}_{1}\right\}^{N_{\mathbf{s}^{(i)}, a_{m}}^{B I N}} \\
& =\left(1-P_{F A}\right)^{N_{r}-N_{\mathbf{s}^{(i)}, a_{m}}^{B I N} P_{M D}^{N_{\mathbf{s}}^{(i)}, a_{m}}},
\end{aligned}
$$

and

$$
\operatorname{Pr}\left\{o_{m}=1 \mid \mathbf{s}^{(i)}, a_{m}\right\}=1-\operatorname{Pr}\left\{o_{m}=0 \mid \mathbf{s}^{(i)}, a_{m}\right\}
$$

where $\left(P_{F A}, P_{M D}\right)$ for the Neyman-Pearson detector is given in (21), and $N_{\mathbf{s}^{(i)}, a_{m}}^{B I N}$ is the actual number of non-zero bins in the $a_{m}$-th column of the VCM when the VCM is in state $\mathbf{s}^{(i)}$. Here, two paths with the same AoD and different AoAs are regarded as two different paths. (24) is obtained because for the AoA directions with no paths in the $a_{m}$-th column of the VCM the detector should declare $\mathcal{H}_{0}$ and for the AoA directions with paths in the $a_{m}$-th column of the VCM the detector should miss to have $o_{m}=0$ (i.e., no non-zero bin in the $a_{m}$-th column is declared). The path complex gain can be estimated based on (18) with a certain estimator such as the maximum likelihood or minimum mean-square-error (MMSE) estimator.

\section{B. Sufficient Statistic}

Fig. 5 depicts the sequence of the operation at slot $k$ composed of the current belief vector $\pi_{k}$, state transition, action, observation, and following reward. In the beginning of slot $k$, the 
information from all the past slots is summarized as a belief vector: ${ }^{\S}$

$$
\boldsymbol{\pi}_{k}=\left[\pi_{k, 1}, \pi_{k, 2}, \cdots, \pi_{k, N}\right]
$$

where $\pi_{k, i}$ is the probability that the state at the beginning of slot $k$ is state $\mathbf{s}^{(i)}$ conditioned on all past pilot beam sequences and feedback information. It is known that the belief vector is a sufficient statistic for the action, i.e., the design of the optimal pilot beam sequence for slot $k$ in our case [23]. The transmitter uses the belief vector to optimally choose the action for slot $k$ from the action space that maximizes the expected reward, and updates the belief vector for the next block based on the new feedback information at the current slot.

\section{The Reward and The Policy}

Depending on the result of channel identification, a reward is gained during the data transmission period. According to the system objective, several reward definitions can be considered. In our case, since the purpose of channel estimation is data transmission, we consider the number of successfully transmitted bits over the total considered period of $T$ slots as the final reward. With the assumption of $\alpha_{(k), l} \stackrel{\text { i.i.d. }}{\sim} \mathcal{C N}\left(0, \xi^{2}\right)$ in (2), the data rate for slot $k$ will roughly be $\log \left(1+N_{p} N_{t} N_{r} \xi^{2} / \sigma_{N}^{2}\right)$ in case of maximal ratio combining (MRC) transmission and reception or $N_{p} \log \left(1+N_{t} N_{r} \xi^{2} / \sigma_{N}^{2}\right)$ in case of spatial multiplexing used at the transmitter, where $N_{p}$ is the number of correctly identified paths. Here, under the assumption of spatial multiplexing on different paths, the number of successfully transmitted bits per slot is proportional to $N_{p}$. Thus, we choose the number of correctly identified paths as the slot reward.

If the state of the VCM at slot $k$ is $\mathbf{s}^{(i)}$, the selected pilot beam sequence at slot $k$ is $\mathbf{a}$, and the transmitter observes the feedback information $\mathbf{o}^{(j)}$, then the immediate reward at slot $k$ can be expressed as

$$
r\left(\mathbf{s}^{(i)}, \mathbf{a}, \mathbf{o}^{(j)}\right)=\sum_{m=1}^{M_{p}} N_{\mathbf{s}^{(i)}, a_{m}}^{B I N} o_{m}^{(j)}
$$

where $o_{m}^{(j)}$ is the $m$-th element of $\mathbf{o}^{(j)}$. Note that the false alarm of the receiver detector does not affect the immediate reward because in this case we have $o_{m}^{(j)}=1$ but $N_{\mathbf{s}^{(i)}, a_{m}}^{B I N}=0$. (However,

${ }^{\S}$ Following the convention, we define the belief vector $\boldsymbol{\pi}_{k}$ prior to the state transition for each slot, as shown in Fig. 5. The belief vector after the state transition can be updated easily based on the state transition probability matrix.

${ }^{\mathbb{q}}$ In case of MRC, we use $r\left(\mathbf{s}^{(i)}, \mathbf{a}, \mathbf{o}^{(j)}\right)=\log \left(1+\left[\sum_{m=1}^{M_{p}} N_{\mathbf{s}^{(i)}, a_{m}}^{B I N} o_{m}^{(j)}\right] N_{t} N_{r} \xi^{2} / \sigma_{N}^{2}\right)$ instead. 
there will be no ACK for the transmitted packet over the slot and the communication resource will be wasted. The false alarm probability $P_{F A}$ of the channel sensor should be determined properly by considering this resource waste.) In the case of miss detection, the opportunity of successful data transmission is lost. Thus, by reducing the miss probability $P_{M D}$ of the channel sensor we can obtain more reward. It is known that the Neyman-Pearson detector minimizes $P_{M D}$ for given $P_{F A}[21]$.

Note that the state at slot $k$ and the feedback information are unknown at the time of action. Hence, we should consider the expected reward [17]. If the VCM state prior to the state transition at slot $k$ is $\mathbf{s}^{(n)}$ and we perform action $\mathbf{a}$, then the immediate expected reward at slot $k$ is given by

$$
\begin{aligned}
R\left(\mathbf{s}^{(n)}, \mathbf{a}\right) & =\sum_{i=1}^{N} p_{n i} \sum_{j=1}^{2^{M_{p}}} \operatorname{Pr}\left\{\mathbf{o}=\mathbf{o}^{(j)} \mid \mathbf{s}^{(i)}, \mathbf{a}\right\} r\left(\mathbf{s}^{(i)}, \mathbf{a}, \mathbf{o}^{(j)}\right) \\
& =\sum_{i=1}^{N} p_{n i} \sum_{j=1}^{2^{M_{p}}} q_{i j}^{\mathbf{a}} \sum_{m=1}^{M_{p}} N_{\mathbf{s}^{(i)}, a_{m}}^{B I N} o_{m}^{(j)},
\end{aligned}
$$

where the state transition from $\mathbf{s}^{(n)}$ to all possible $\mathbf{s}^{(i)}$ within the slot is captured by $\sum_{i=1}^{N} p_{n i}(\cdot)$. If the belief vector $\boldsymbol{\pi}_{k}$ is given at the beginning of slot $k$ and $S_{k}$ is the random variable representing the state at the beginning of the slot, then the (average) immediate expected reward for action a at slot $k$ can be expressed as

$$
\begin{aligned}
\mathcal{R}\left(\boldsymbol{\pi}_{k}, \mathbf{a}\right) & =\mathbb{E}\left\{R\left(S_{k}, \mathbf{a}\right) \mid \boldsymbol{\pi}_{k}\right\} \\
& =\sum_{i=1}^{N} \pi_{k, i} R\left(\mathbf{s}^{(i)}, \mathbf{a}\right)=\left\langle\mathbf{R}(\mathbf{a}), \boldsymbol{\pi}_{k}\right\rangle,
\end{aligned}
$$

where $\mathbf{R}(\mathbf{a}):=\left[R\left(\mathbf{s}^{(1)}, \mathbf{a}\right), R\left(\mathbf{s}^{(2)}, \mathbf{a}\right), \cdots, R\left(\mathbf{s}^{(N)}, \mathbf{a}\right)\right]$, and $\langle\cdot, \cdot\rangle$ denotes the inner product operation.

In the POMDP framework, a policy $\delta$ is defined as a sequence of functions that maps the belief vector to an action for each slot $[23,24]$. The optimal policy is one that maximizes the total immediate expected reward accumulated over the considered total time of $T$ slots ${ }^{\|}$when the initial belief vector at the beginning of the transmission is given as $\pi_{1}$. That is, the optimal

"Such a formulation is called a finite-horizon POMDP. The considered formulation can be modified to the infinite-horizon case. 
policy $\delta^{*}$ is expressed as

$$
\delta^{*}=\underset{\delta}{\arg \max } \mathbb{E}_{\delta}\left[\sum_{k=1}^{T} R\left(S_{k}, \mathbf{a}_{k}\right) \mid \boldsymbol{\pi}_{1}\right],
$$

where $\mathbf{a}_{k}$ is the action vector for slot $k$, and $\mathbb{E}_{\delta}$ is the conditional expectation when the policy $\delta$ is given.

\section{Optimal and Suboptimal Strategies for Training Beam Design}

\section{A. The Optimal Strategy}

Under the proposed POMDP formulation, the optimal training beam sequence design for maximizing the accumulated data rate over $T$ slots is equivalent to the problem of finding the optimal policy satisfying (30). To solve this problem we define the optimal value function $V\left(\boldsymbol{\pi}_{1}\right)$ as the maximum total expected reward obtained by the optimal policy $\delta^{*}$ when the initial belief vector $\pi_{1}$ is given at $k=1$ :

$$
V\left(\boldsymbol{\pi}_{1}\right)=\mathbb{E}_{\delta^{*}}\left[\sum_{k=1}^{T} R\left(S_{k}, \mathbf{a}_{k}\right) \mid \boldsymbol{\pi}_{1}\right]
$$

Next consider $V^{k}\left(\boldsymbol{\pi}_{k}\right)$ defined as the maximum remaining expected reward that can be obtained from slot $k$ to slot $T$ when a belief vector $\pi_{k}$ is given at the beginning of slot $k$. By separating slot $k$ and the remaining slots, $V^{k}\left(\boldsymbol{\pi}_{k}\right)$ can be decomposed as [25]

$$
V^{k}\left(\boldsymbol{\pi}_{k}\right)=\max _{\mathbf{a}_{k}}\left\{\left\langle\mathbf{R}\left(\mathbf{a}_{k}\right), \boldsymbol{\pi}_{k}\right\rangle+\sum_{j=1}^{2^{M_{p}}} V^{k+1}\left(\mathcal{T}\left(\boldsymbol{\pi}_{k} \mid \mathbf{a}_{k}, \mathbf{o}^{(j)}\right)\right) \gamma\left(\mathbf{o}^{(j)} \mid \boldsymbol{\pi}_{k}, \mathbf{a}_{k}\right)\right\}
$$

where $\gamma\left(\mathbf{o}^{(j)} \mid \boldsymbol{\pi}_{k}, \mathbf{a}_{k}\right)=\sum_{i=1}^{N} q_{i j}^{\mathbf{a}_{k}} \sum_{n=1}^{N} \pi_{k, n} p_{n i}$ is the probability that $\mathbf{o}^{(j)}$ is observed given belief vector $\boldsymbol{\pi}_{k}$ and action $\mathbf{a}_{k}$ for slot $k$, and $\mathcal{T}\left(\boldsymbol{\pi}_{k} \mid \mathbf{a}_{k}, \mathbf{o}^{(j)}\right)$ is the updated belief vector from $\boldsymbol{\pi}_{k}$ at slot $k$ for slot $k+1$ after taking action $\mathbf{a}_{k}$ and observing $\mathbf{o}^{(j)}$. Here, $\mathcal{T}\left(\boldsymbol{\pi}_{k} \mid \mathbf{a}_{k}, \mathbf{o}^{(j)}\right)$ can be computed using Bayes's rule as [23, 24]

$$
\mathcal{T}\left(\boldsymbol{\pi}_{k} \mid \mathbf{a}_{k}, \mathbf{o}^{(j)}\right)=\boldsymbol{\pi}_{k+1}=\left[\pi_{k+1,1}, \pi_{k+1,2}, \cdots, \pi_{k+1, N}\right],
$$

where

$$
\pi_{k+1, i}=\operatorname{Pr}\left\{\mathbf{s}_{k}=\mathbf{s}^{(i)} \mid \boldsymbol{\pi}_{k}, \mathbf{a}_{k}, \mathbf{o}^{(j)}\right\}=\frac{q_{i j}^{\mathbf{a}_{k}} \sum_{n=1}^{N} \pi_{k, n} p_{n i}}{\sum_{i=1}^{N} q_{i j}^{\mathbf{a}_{k}} \sum_{n=1}^{N} \pi_{k, n} p_{n i}}, i=1, \cdots, N .
$$


The first term in the left-hand side (LHS) of (32) is the immediate expected reward for slot $k$ and the second term is the the maximum remaining expected reward from slot $k+1$. As shown in (32), the selected pilot beam sequence $\mathbf{a}_{k}$ for slot $k$ affects not only the immediate expected reward at slot $k$ but also the maximum remaining reward that can be obtained from slot $k+1$. Hence, by considering the expected reward of the future slots, the performance can be improved over only considering the immediate reward at each slot, i.e., the first term in the LHS of (32).

There exist several known algorithms to obtain the optimal policy $\delta^{*}$ over the considered transmission period $k=[1,2, \cdots, T]$ based on (32) when the state transition probability $\mathbf{P}$, reward, and observation and action spaces are given [23, 24, 26]. For example, point-based POMDP value iteration algorithms are proposed for efficiency and low-complexity [27-29]. However, as the number of states and the size of the action space increase, it requires high computational complexity to obtain the optimal policy for the POMDP problem even with these point-based POMDP value iteration algorithms. Thus, to reduce the complexity, we can alternatively use a suboptimal greedy policy that considers only the immediate expected reward at each slot, i.e., the first term in the LHS of (32). However, in large mmWave MIMO channels with large transmit antenna arrays, even this greedy policy requires high computational complexity due to the huge numbers of states and actions. Thus, in the next subsection we propose a way to implement the greedy policy with significantly reduced complexity making the proposed POMDP-based training beam design for large mmWave MIMO channels practical.

Remark 1: The optimal policy or a suboptimal policy can be computed off-line once the channel dynamic and other parameters are given, and the computed policy can be stored beforehand. Then, in actual transmission, we start from $k=1$ with $\pi_{1}$, and repeat action and observation until slot $T$. The complexity of this actual operation is insignificant in general. This is one of the main advantages of the proposed training beam design approach.

\section{B. The Proposed Greedy Approach with a Reduced Sufficient Statistic}

Although a policy for the POMDP problem can be obtained off-line as mentioned in the above, it is prohibitive in the case of large mmWave MIMO channels since the numbers of states and actions grow as $N_{t}^{L}$ and $\left(\begin{array}{l}N_{t} \\ M_{p}\end{array}\right)$, respectively, in the standard formulation in the previous sections. For example, when $N_{t}=64, M_{p}=10$, and $L=3$, the size of the action space is $1.5 \times 10^{11}$ and 
the number of states is 262,144 . The action space size of $1.5 \times 10^{11}$ combined with the state size 262,144 makes solving the POMDP problem prohibitive. Thus, we here focus on the greedy policy that considers the first term in the LHS of (32) (i.e., the term in (29)) and its fast solution by introducing a reduced sufficient statistic that has only $N_{t} L$ elements.

Proposition 1: With Assumption 1, let $\omega_{k, l, i}$ be the conditional probability (given the pilot sequences and feedback information of all past slots) that the $l$-th path is in the $i$-th column of the VCM at the beginning of slot $k$ for $i=1, \cdots, N_{t}$. Then,

$$
\boldsymbol{\omega}_{k}=\left[\omega_{k, 1,1}, \omega_{k, 1,2}, \cdots, \omega_{k, 1, N_{t}}, \omega_{k, 2,1}, \cdots, \omega_{k, L, N_{t}}\right]
$$

is a sufficient statistic to design the optimal pilot sequence at slot $k$. Here, $\sum_{l i} \omega_{k, l, i}=L$.

Proof: To prove this statement, we only need to show that $\boldsymbol{\pi}_{k}$ can be expressed by the elements of $\boldsymbol{\omega}_{k}$ since $\boldsymbol{\pi}_{k}$ is a sufficient statistic. Consider $\pi_{k, n}$ for each enumerated state $\mathbf{s}^{(n)}$ in $\mathcal{S}$. The state $\mathbf{s}^{(n)}$ can be represented by $\left(i_{1}, i_{2}, \cdots, i_{L}\right)$ that means that the $l$-th path is located in the $i_{l}$-th column of the VCM, $l=1, \cdots, L$ (see Section II-B.2). Then, we have

$$
\begin{aligned}
\pi_{k, n} & =\operatorname{Pr}\left\{S_{k}=\left(i_{1}, i_{2}, \cdots, i_{L}\right) \mid \mathcal{I}_{k}\right\} \\
& =\operatorname{Pr}\left\{i_{1} \mid \mathcal{I}_{k}\right\} \times \cdots \times \operatorname{Pr}\left\{i_{L} \mid \mathcal{I}_{k}\right\} \\
& =\omega_{k, 1, i_{1}} \omega_{k, 2, i_{2}} \cdots \omega_{k, L, i_{L}}
\end{aligned}
$$

where $\mathcal{I}_{k}$ represents the information from all the previous slots before slot $k$, and the second equality follows from Assumption 1 of independent paths.

Now we propose a greedy training beam sequence design algorithm using the reduced belief vector $\boldsymbol{\omega}_{k}$. If the reduced belief vector $\boldsymbol{\omega}_{k}$ is given at the beginning of slot $k$ and the pilot beam sequence $\mathbf{a}$ is chosen for the slot, then the immediate expected reward $\mathcal{R}\left(\boldsymbol{\pi}_{k}, \mathbf{a}\right)\left(=\left\langle\mathbf{R}(\mathbf{a}), \boldsymbol{\pi}_{k}\right\rangle\right)$ at slot $k$ in (29) can be expressed in terms of $\boldsymbol{\omega}_{k}$ as

$$
\mathcal{R}^{\prime}\left(\boldsymbol{\omega}_{k}, \mathbf{a}\right)=\sum_{l=1}^{L}\left(\sum_{m=1}^{M_{p}} \operatorname{Pr}\left\{o_{m}=1 \mid \Omega_{k}\left(l, a_{m}\right)=1\right\} \sum_{n=1}^{N_{t}} \omega_{k, l, n} p_{n, a_{m}}\right)
$$

due to the independence of the paths, where the binary random variable $\Omega_{k}\left(l, a_{m}\right)$ is defined as

$$
\Omega_{k}\left(l, a_{m}\right)= \begin{cases}1, & \text { if the } l \text {-th path is located at the } a_{m} \text {-th column of the VCM at slot } k, \\ 0, & \text { otherwise. }\end{cases}
$$


Here, $\sum_{n=1}^{N_{t}} \omega_{k, l, n} p_{n, a_{m}}$ is the probability that the $l$-th path is in the $a_{m}$-th column of the VCM at slot $k$ after the state transition within the slot, and $\operatorname{Pr}\left\{o_{m}=1 \mid \Omega_{k}\left(l, a_{m}\right)=1\right\}$ is the probability that the receiver detects the $l$-th path successfully when the $l$-th path is in the $a_{m}$-th column of the VCM. $\operatorname{Pr}\left\{o_{m}=1 \mid \Omega_{k}\left(l, a_{m}\right)=1\right\}$ in (36) is related to the miss detection probability of the receiver detector. To obtain a tractable expression for the immediate expected reward, we assume that we have a reasonable path sensing SNR $N_{t} N_{r} P_{t} \xi^{2} / \sigma_{N}^{2}$ and the miss detection probability $P_{M D}$ of the receiver detector is zero, i.e., $\operatorname{Pr}\left\{o_{m}=1 \mid \Omega_{k}\left(l, a_{m}\right)=1\right\}=1$. Then, (36) can be rewritten as

$$
\begin{aligned}
\mathcal{R}^{\prime}\left(\boldsymbol{\omega}_{k}, \mathbf{a}\right) & =\sum_{l=1}^{L} \sum_{m=1}^{M_{p}} \sum_{n=1}^{N_{t}} \omega_{k, l, n} p_{n, a_{m}} \\
& =\sum_{l=1}^{L} \sum_{n=1}^{N_{t}} \omega_{k, l, n} \sum_{m=1}^{M_{p}} p_{n, a_{m}} \\
& =[\underbrace{\sum_{m=1}^{M_{p}} p_{1, a_{m}}, \cdots, \sum_{m=1}^{M_{p}} p_{N_{t}, a_{m}}}_{1 \mathrm{st} \text { path }}, \cdots, \underbrace{\sum_{m=1}^{M_{p}} p_{1, a_{m}}, \cdots, \sum_{m=1}^{M_{p}} p_{N_{t}, a_{m}}}_{L-1 \mathrm{th} \text { path }}] \boldsymbol{\omega}_{k} \\
& \stackrel{(a)}{=} \sum_{m \in \mathbf{a}} \mathbf{P}^{\prime}(m,:) \boldsymbol{\omega}_{k}
\end{aligned}
$$

where $\mathbf{P}^{\prime}:=[\underbrace{\mathbf{P}^{T}}_{1 \text { st }}, \mathbf{P}^{T}, \cdots, \underbrace{\mathbf{P}^{T}}_{L \text {-th }}]$ with $\mathbf{P}$ defined in (9), and $\mathbf{P}^{\prime}(m,:)$ denotes the $m$-th row of $\mathbf{P}^{\prime}$. (In step (a), the summation went out of the bracket.) Therefore, the greedy training beam sequence design problem of choosing a that maximize the immediate expected reward at slot $k$ reduces to choosing a such that

$$
\mathbf{a}^{*}=\underset{\mathbf{a}}{\arg \max } \sum_{m \in \mathbf{a}} \mathbf{P}^{\prime}(m,:) \boldsymbol{\omega}_{k}
$$

Thus, for the optimal choice of the training beam sequence for the greedy policy, from (38), we only need to find the set of the indices that correspond to the largest $M_{p}$ values in the $N_{t} \times 1$ vector $\mathbf{P}^{\prime} \boldsymbol{\omega}_{k}$, which can be obtained by $N_{t}^{2} L$ real multiplications. Note that in the proposed solution we do not need to consider $\left(\begin{array}{c}N_{t} \\ M_{p}\end{array}\right)$ complexity for a, but one time sorting of an $N_{t} \times 1$ vector is enough! 
At the end of slot $k$, the transmitter updates the reduced belief vector from $\boldsymbol{\omega}_{k}$ to $\boldsymbol{\omega}_{k+1}$ based on the selected pilot beam sequence and the observed feedback information at the current slot. This update process is given in the following proposition.

Proposition 2: With the action vector a and observation $\mathbf{o}^{(j)}$ for slot $k$, the updated reduced sufficient statistic is given by

$$
\mathcal{T}\left(\boldsymbol{\omega}_{k} \mid \mathbf{a}, \mathbf{o}^{(j)}\right):=\boldsymbol{\omega}_{k+1}=\left[\omega_{k+1,1,1}, \omega_{k+1,1,2}, \cdots, \omega_{k+1, L, N_{t}}\right]
$$

where each element of $\boldsymbol{\omega}_{k+1}$ is obtained by Bayes's rule as

$$
\omega_{k+1, l, i}=\frac{q_{i j l}^{\mathbf{a}} \sum_{n=1}^{N_{t}} \omega_{k, l, n} p_{n i}}{\sum_{i=1}^{N_{t}} q_{i j l}^{\prime \mathbf{a}} \sum_{n=1}^{N_{t}} \omega_{k, l, n} p_{n i}},
$$

and $q_{i j l}^{\text {a }}$ is the probability that the $j$-th feedback information $\mathbf{o}^{(j)}$ is observed conditioned on that the $l$-th path is located in the $i$-th column of the VCM after state transition within the slot and the pilot beam sequence a is transmitted. The quantity $q_{i j l}^{\text {a }}$ is given by

$$
q_{i j l}^{\prime \mathbf{a}}=\prod_{m=1}^{M_{p}} \operatorname{Pr}\left\{o_{m}=o_{m}^{(j)} \mid \Omega_{k+1}(l, i)=1, \mathbf{a}, \boldsymbol{\omega}_{k}\right\} .
$$

where $\operatorname{Pr}\left\{o_{m}^{(j)}=1 \mid \Omega_{k+1}(l, i)=1, \mathbf{a}, \boldsymbol{\omega}_{k}\right\}=1-\operatorname{Pr}\left\{o_{m}^{(j)}=0 \mid \Omega_{k+1}(l, i)=1, \mathbf{a}, \boldsymbol{\omega}_{k}\right\}$, and

$$
\begin{aligned}
\operatorname{Pr}\left\{o_{m}^{(j)}=\right. & \left.0 \mid \Omega_{k+1}(l, i)=1, \mathbf{a}, \boldsymbol{\omega}_{k}\right\} \\
= & \begin{cases}P_{M D}^{\prime}, & \text { if } a_{m}=i, \\
\prod_{s=1, s \neq l}^{L}\left(\sum_{t=1}^{N_{t}} \omega_{k, s, t}\left(1-p_{t a_{m}}\right)\right)\left(1-P_{F A}\right) & \\
\quad+\left(1-\prod_{s=1, s \neq l}^{L}\left(\sum_{t=1}^{N_{t}} \omega_{k, s, t}\left(1-p_{t a_{m}}\right)\right)\right) P_{M D}^{\prime}, & \text { if } a_{m} \neq i .\end{cases}
\end{aligned}
$$

Here, $P_{M D}^{\prime}$ is the probability of miss detection at the channel sensor when at least one path exists in the sensed column of the VCM.

Proof: Applying Bayes's formula, we express $\omega_{k+1, l, i}$ in (39) as

$$
\begin{aligned}
\omega_{k+1, l, i} & =\operatorname{Pr}\left\{\Omega_{k+1}(l, i)=1 \mid \mathbf{o}^{(j)}, \mathbf{a}, \boldsymbol{\omega}_{k}\right\} \\
& =\frac{\operatorname{Pr}\left\{\Omega_{k+1}(l, i)=1, \mathbf{o}^{(j)} \mid \mathbf{a}, \boldsymbol{\omega}_{k}\right\}}{\sum_{i=1}^{N_{t}} \operatorname{Pr}\left\{\Omega_{k+1}(l, i)=1, \mathbf{o}^{(j)} \mid \mathbf{a}, \boldsymbol{\omega}_{k}\right\}}
\end{aligned}
$$


The numerator of (43) is expressed as follows:

$$
\begin{aligned}
\operatorname{Pr}\left\{\Omega_{k+1}(l, i)=1, \mathbf{o}=\mathbf{o}^{(j)} \mid \mathbf{a}, \boldsymbol{\omega}_{k}\right\} \\
=\sum_{n=1}^{N_{t}} \operatorname{Pr}\left\{\Omega_{k}(l, n)=1, \Omega_{k+1}(l, i)=1, \mathbf{o}=\mathbf{o}^{(j)} \mid \mathbf{a}, \boldsymbol{\omega}_{k}\right\} \\
=\sum_{n=1}^{N_{t}} \operatorname{Pr}\left\{\Omega_{k}(l, n)=1 \mid \mathbf{a}, \boldsymbol{\omega}_{k}\right\} \cdot \operatorname{Pr}\left\{\Omega_{k+1}(l, i)=1, \mathbf{o}=\mathbf{o}^{(j)} \mid \Omega_{k}(l, n)=1, \mathbf{a}, \boldsymbol{\omega}_{k}\right\} \\
=\sum_{n=1}^{N_{t}} \operatorname{Pr}\left\{\Omega_{k}(l, n)=1 \mid \mathbf{a}, \boldsymbol{\omega}_{k}\right\} \cdot \operatorname{Pr}\left\{\Omega_{k+1}(l, i)=1 \mid \Omega_{k}(l, n)=1, \mathbf{a}, \boldsymbol{\omega}_{k}\right\} \\
\quad \cdot \operatorname{Pr}\left\{\mathbf{o}=\mathbf{o}^{(j)} \mid \Omega_{k+1}(l, i)=1, \Omega_{k}(l, n)=1, \mathbf{a}, \boldsymbol{\omega}_{k}\right\} \\
=\sum_{n=1}^{N_{t}} \omega_{k, l, n} \cdot p_{n i} \cdot \operatorname{Pr}\left\{\mathbf{o}=\mathbf{o}^{(j)} \mid \Omega_{k+1}(l, i)=1, \mathbf{a}, \boldsymbol{\omega}_{k}\right\},
\end{aligned}
$$

where the last step follows by the definitions of $\omega_{k, l, n}$ and $p_{n i}$ and the Markovian assumption on the state transition. Denoting $\operatorname{Pr}\left\{\mathbf{o}=\mathbf{o}^{(j)} \mid \Omega_{k+1}(l, i)=1, \mathbf{a}, \boldsymbol{\omega}_{k}\right\}$ by $q_{i j l}^{\prime \text { a }}$, we have (40).

As in deriving (22), once the state is given, the remaining randomness is the receiver noise. Thus, by the assumption of independent receiver noise over symbol time, we have

$$
\begin{aligned}
q_{i j l}^{\prime \mathbf{a}} & :=\operatorname{Pr}\left\{\mathbf{o}=\mathbf{o}^{(j)} \mid \Omega_{k+1}(l, i)=1, \mathbf{a}, \boldsymbol{\omega}_{k}\right\} \\
& =\prod_{m=1}^{M_{p}} \operatorname{Pr}\left\{o_{m}=o_{m}^{(j)} \mid \Omega_{k+1}(l, i)=1, \mathbf{a}, \boldsymbol{\omega}_{k}\right\} .
\end{aligned}
$$

If the $l$-th path is in the $i$-th column of the VCM and the $i$-th column of the VCM is chosen to be sensed by the $m$-th pilot beam in the slot (i.e., $a_{m}=i$ ), the probability that $o_{m}^{(j)}=0$ is observed is equal to the miss detection probability $P_{M D}^{\prime}$ of the channel sensor. This explains the first part of (42). If the $m$-th pilot beam senses a column that does not contain the $l$-th path located at the $i$-th column of the VCM (i.e., $a_{m} \neq i$ ), then the probability that $o_{m}^{(j)}=0$ is observed is equal to

$$
\prod_{s=1, s \neq l}^{L}\left(\sum_{t=1}^{N_{t}} \omega_{k, s, t}\left(1-p_{t a_{m}}\right)\right)\left(1-P_{F A}\right)+\left(1-\prod_{s=1, s \neq l}^{L}\left(\sum_{t=1}^{N_{t}} \omega_{k, s, t}\left(1-p_{t a_{m}}\right)\right)\right) P_{M D}^{\prime}
$$

The first term in (46) is the probability that no other path than the $l$-th path is located in the $a_{m}$-th column of the VCM and a false alarm does not occur, and the second term in (46) is the probability that at least one path other than the $l$-th path exists in the $a_{m}$-th column of the VCM but the channel sensor misses this path. This explains the second part of (42). 
For simplicity, we use $P_{M D}$ in the single path case instead of $P_{M D}^{\prime}$ neglecting the case that there can exist more than one path in the sensed column of the VCM. However, this will have a negligible impact on the performance because the event that more than one path are located in the same column of the VCM will rarely occur due to the sparsity of large mm-Wave MIMO channels. Note that the action vector computation and the belief vector update required for the proposed greedy algorithm based on the reduced sufficient statistic can be run with insignificant computational complexity.

\section{Extensions}

Multi-resolution: Until now we have assumed that the transmitter picks one column of $\mathbf{A}_{T}$ as the pilot beam at each pilot symbol time and search one column of the VCM at each pilot symbol time, i.e., $\mathbf{x}_{n} \in\left\{\sqrt{P_{t}} \mathbf{a}_{T X}\left(\tilde{\theta}_{1}^{t}\right), \cdots, \sqrt{P_{t}} \mathbf{a}_{T X}\left(\tilde{\theta}_{N_{t}}^{t}\right)\right\}$. This assumption can be relaxed depending on the path SNR. Recall that the path SNR is given by $N_{t} N_{r} P_{t} \xi^{2} / \sigma_{N}^{2}$, where $\xi^{2}$ is the path gain square and $\sigma_{N}^{2}$ is the noise variance. When the path SNR is sufficiently high, we can use a superposed pilot beam at each symbol time, i.e.,

$$
\mathbf{x}_{n} \in\left\{\sum_{i=1}^{N_{a}} \sqrt{\frac{P_{t}}{N_{a}}} \mathbf{a}_{T X}\left(\tilde{\theta}_{i}^{t}\right), \sum_{i=N_{a}+1}^{2 N_{a}} \sqrt{\frac{P_{t}}{N_{a}}} \mathbf{a}_{T X}\left(\tilde{\theta}_{i}^{t}\right), \cdots, \sum_{i=N_{t}-N_{a}+1}^{N_{t}} \sqrt{\frac{P_{t}}{N_{a}}} \mathbf{a}_{T X}\left(\tilde{\theta}_{i}^{t}\right)\right\},
$$

where $N_{a}$ consecutive columns of $\mathbf{A}_{T}$ are superposed as one pilot beam for a symbol time under the assumption that $N_{a}$ is a divisor of $N_{t}$. This is equivalent to effectively making the search grid in the VCM small and shortening the initial lock-in time. The corresponding state transition probability matrix can be obtained from the original state transition matrix $\mathbf{P}$. Once the nonzero paths in the smaller grid are detected, we can switch to the original high-resolution narrow pilot beam by properly assigning the belief vector. Here, $N_{a}$ should be determined appropriately so that $N_{t} N_{r} P_{t} \xi^{2} /\left(N_{a} \sigma_{N}^{2}\right)$ is high enough to make the probability of miss detection by each superposed pilot beam small.

2-dimensional search with limited receiver beamforming: We have assumed that the receiver has a filter bank that searches all AoA directions. This requires $N_{r}$ RF chains at the receiver. When the receiver has one RF chain, typically the beamforming is implemented at the antenna array. That is, the antenna array has multiple receive beamforming directions and switching is performed among the receive beamforming directions. In this case, the proposed POMDP framework can be extended to search the non-zero paths in a truly 2-dimensional grid. 
The mult-user case: Until now we have considered the optimal training beam design for channel estimation and tracking via POMDP in single-user MIMO systems. We can apply our POMDP formulation to downlink multi-user MIMO channels with simple modifications. The MIMO channel with multiple users can be considered as the MIMO channel with more paths. In the multi-user case, the transmitter needs to know which user each path belongs to. This identification can be delivered in feedback information from users, and the POMDP formulation with this additional feedback information can be used in the multi-user MIMO case.

\section{NumericAl RESUlts}

In this section, we provide some numerical results to evaluate the performance of the proposed pilot design and channel estimation method. Throughout the simulation, we fixed the number of paths as $L=2$, the path gain $\xi^{2}=1$, and the noise power $\sigma_{N}^{2}=1$; set the transmit power $P_{t}$ in each case so that the path SNR $N_{t} N_{r} P_{t} \xi^{2} / \sigma_{N}^{2}$ is $20 \mathrm{~dB}$; and used a Neyman-Pearson detector for the channel sensor at the receiver. We assumed that each path behaves according to the state transition probability matrix (11) under Assumption 1.

First, we considered a small MIMO system with $N_{t}=8, N_{r}=4$, and $M_{p}=4$ so that the optimal policy can be computed with reasonable complexity. Here, we set the false alarm probability of the channel sensor for each AoA direction as $P_{F A}=0.05$, and $B=1$ and $\lambda=0$ for $\mathbf{P}_{\beta, \lambda}^{B}$ in (11), and considered two different values of $\beta$ : $\beta=0.5$ (slow fading) and $\beta=0.9$ (fast fading). Fig. 6 shows the performance of three methods in this case: the optimal POMDP strategy, the greedy POMDP strategy and a random selection strategy that selects $M_{p}$ pilot beams randomly at each slot. The $y$-axis shows the averaged per-slot reward, i.e., the averaged number of detected paths for each slot. (The per-slot reward shown in the figure is the average over 100,000 realizations of the specified Markov random processes of length 10 slots.) We first tested the initial lock-in behavior of the three methods. Here, assuming no a priori state information in the beginning, we set all elements of the initial belief vector to be equal. Fig. 6 (a) shows the initial lock-in behavior in this case. On the other hand, Fig. 6 (b) shows the channel tracking behavior of the three methods. Here, we assumed the exact state knowledge at the first slot. It is seen that the optimal POMDP method and the greedy POMDP method significantly outperform the random selection strategy and the performance difference between the optimal strategy and the greedy strategy is not significant. It is also seen that as expected, the channel 


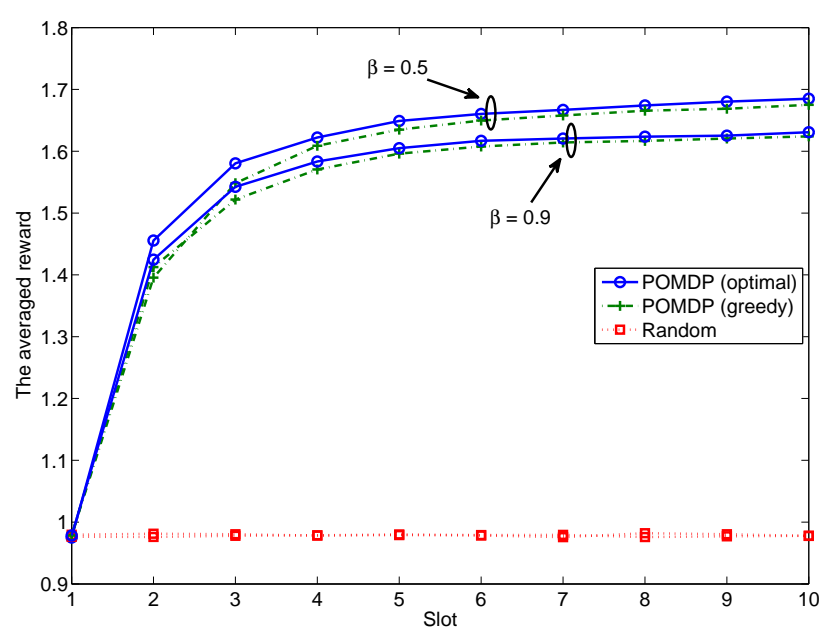

(a)

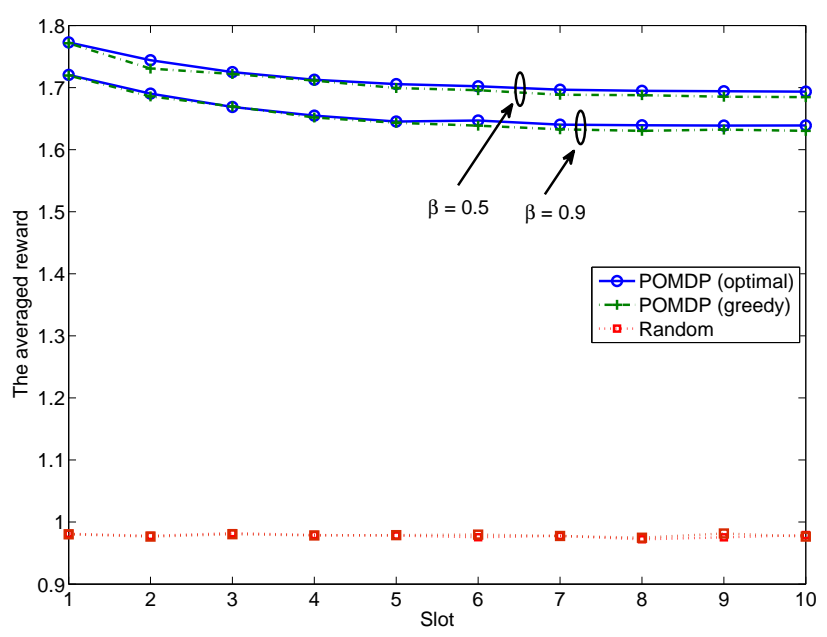

(b)

Fig. 6

AVERAGED PER-SLOt REWARd With RESPECt to time $\left(N_{t}=8, N_{r}=4, M_{p}=4, L=2, \lambda=0, M_{s}=10\right)$

estimation and tracking performance is better at $\beta=0.5$ than at $\beta=0.9$.

Second, we considered a larger MIMO system with $N_{t}=16, N_{r}=4, M_{p}=6$, and $M_{s}=30$ for which the complexity of computing the optimal policy is already high. Here, we considered three pilot beam design and channel estimation methods: the exact greedy POMDP method, the greedy POMDP method with the proposed reduced-size sufficient statistic, and a heuristic 


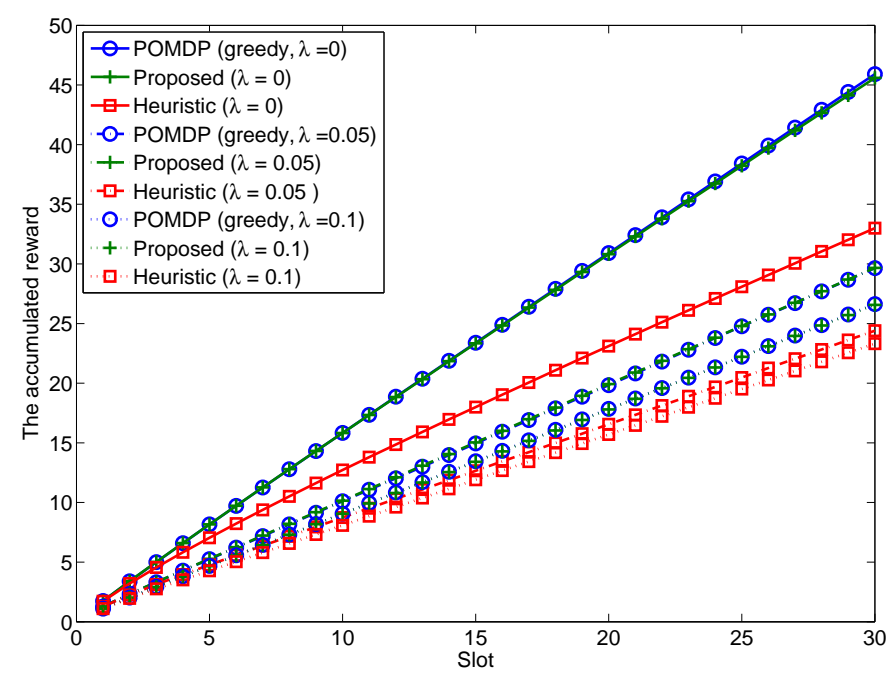

Fig. 7

Accumulated ReWARD With Resect to time $\left(N_{t}=16, N_{r}=4, M_{p}=6, L=2, \beta=0.5, M_{s}=30\right)$

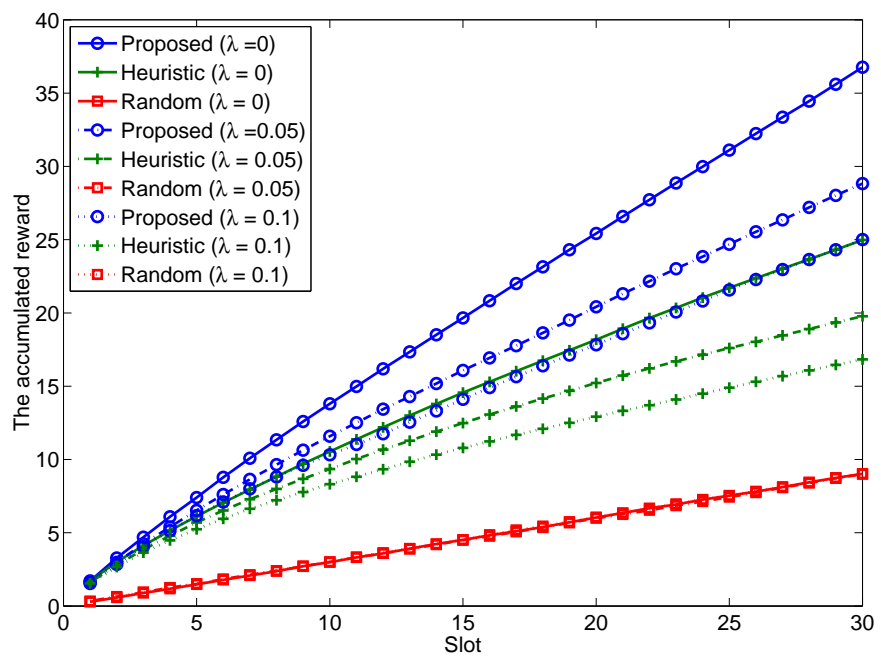

Fig. 8

Accumulated Reward with Resect to time $\left(N_{t}=64, N_{r}=16, M_{p}=10, L=2, \beta=0.5, M_{s}=30\right)$

method. For the state transition probability matrix, we used $\mathbf{P}_{\lambda, \beta}^{B}$ in (11) with $B=2, \beta=0.5$ and varied $\lambda$. We set the false alarm probability of the channel sensor as $P_{F A}=0.05$. For the 
heuristic method, 3 pilot symbol times out of $M_{p}=6$ pilot symbol times per slot are used to track one path and the remaining 3 pilot symbol times are used to track the other path based on the detection result of the previous slot. The heuristic method operates as follows. If a path is detected successively at a slot, the pilot beams of the next slot are adaptively determined to track the path by considering the probability of the path's movement based on the state transition probability matrix. If not, the pilot beams are unchanged for the next slot. Fig. 7 shows the averaged accumulated reward of the three methods with respect to time under the assumption that the initial state is known. The performance in the figure is the average over 10,000 realizations of the specified Markov process of length 30 slots. Recall that in the proposed reduced-complexity greedy method, the exact immediate reward (36) is approximated by (37). It is seen that the impact of this approximation is negligible and the proposed reduced-complexity greedy POMDP method performs as well as the exact greedy POMDP method. It is also seen that the two POMDP-based greedy methods outperforms the heuristic method that still incorporates the state transition in a simple manner.

Next, we considered a large MIMO channel with $N_{t}=64, N_{r}=16$, and $M_{p}=10$. Here, we set $B=8, \beta=0.5$ and varied $\lambda$ for $\mathbf{P}_{\beta, \lambda}^{B}$. In this case, we only considered the proposed reduced-complexity greedy POMDP method and the simple heuristic method that have feasible complexity. Due to the large $N_{r}$, we set the false alarm probability of the channel sensor as $P_{F A}=0.01$ in this case. (If the per-AoA-direction false alarm probability is not sufficiently small in case of large $N_{r}$, the overall false alarm probability combining the sensing results of all the AoA directions is too high.) Fig. 8 shows the accumulated reward performance (with a known initial state) with respect to time (averaged over 500 realizations) in this case. Similar behavior is observed as in Fig. 7. Fig. 9 shows the tracking performance in a single realization of the Markov channel process of length 30 slots. In Fig. 9, the green and blue stars indicate the positions of the two paths at each slot, and $\square$ 's indicate the pilot beam indices at each slot, and $\circ$ 's and $\times$ 's indicate the feedback information of path presence and path absence, respectively, in the sensed index. It is seen that the proposed method tracks the two paths successively, whereas the heuristic strategy is ineffective. In the case of the heuristic method, it is difficult to track the path if the path is missed in the previous block, and it is not possible to distinguish a false alarm. However, the proposed POMDP method tracks the paths in each slot by updating 


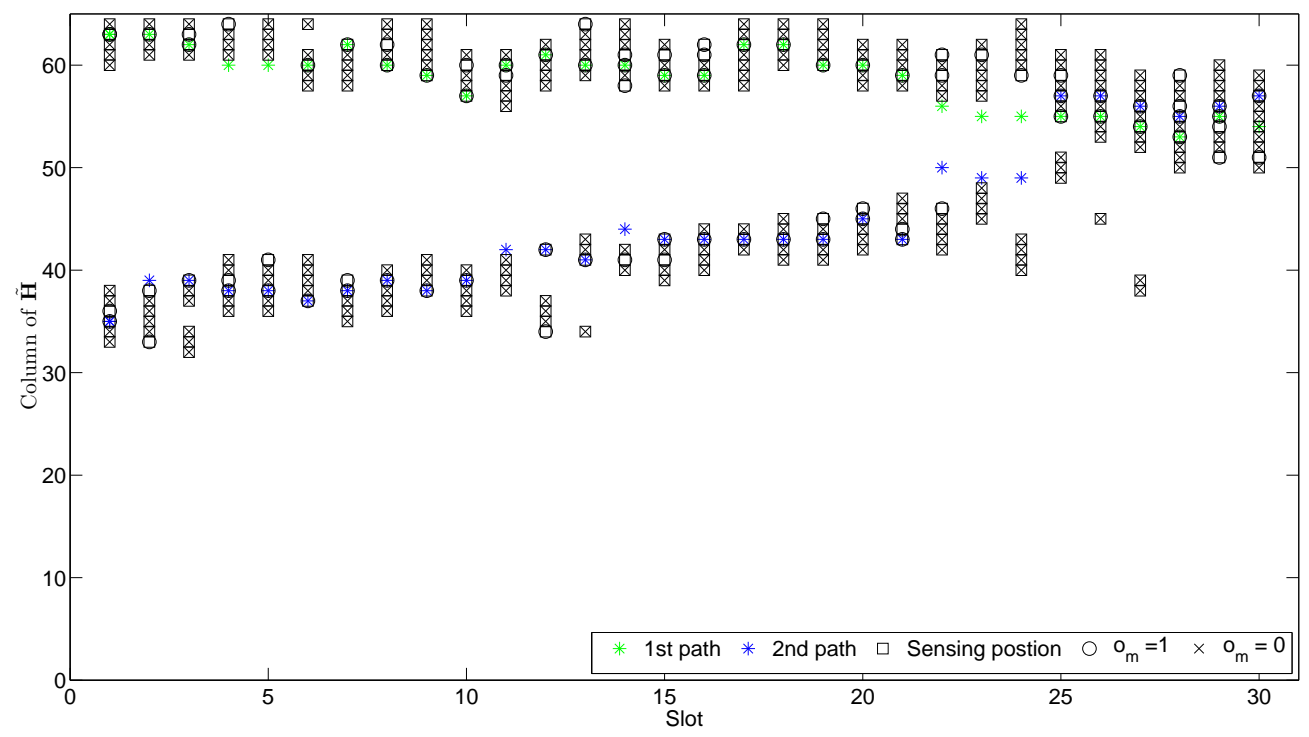

(a)

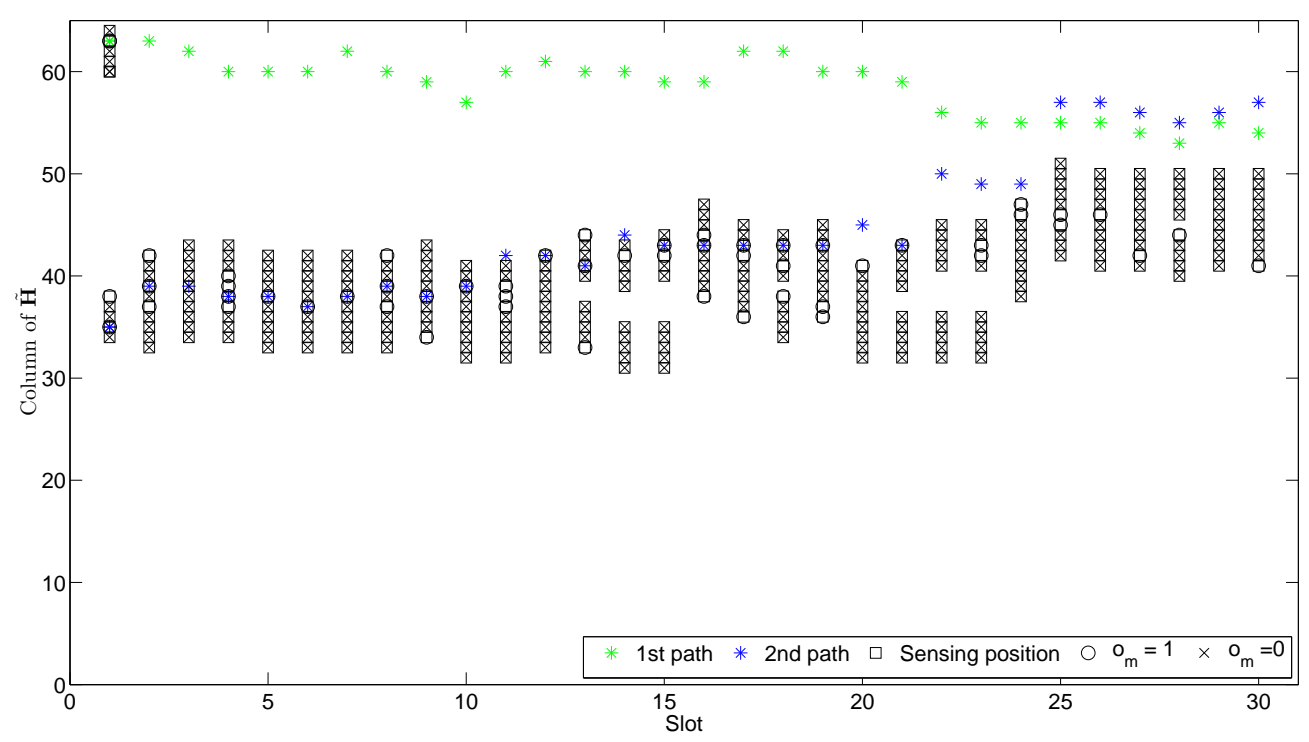

(b)

Fig. 9

The tracking Process in the MiMO Channel $\left(N_{t}=64, N_{r}=16, M_{p}=10, L=2, \lambda=0\right.$ ) (A): The PROPOSED STRATEGY AND (B) : THE HEURISTIC STRATEgY. 
the reduced-size belief vector based on the history, and the false alarm can be handled in the updating process of the reduced-size belief vector. Thus, the proposed POMDP-based method is much more effective in sparse large MIMO channel tracking.

\section{CONCLUSION}

We have considered the adaptive training beam sequence design problem for large sparse mmWave MIMO channels. By imposing a Markov random walk assumption on the paths' movement, we have formulated the adaptive training beam sequence design problem as a POMDP problem. Under this POMDP framework, we have obtained optimal and suboptimal strategies for adaptive training beam sequence design for large sparse mmWave MIMO channels. Furthermore, we have derived a fast greedy POMDP algorithm for this problem with significantly reduced complexity for practical implementation by proposing a new sufficient statistic for the decision process. We have considered several extensions of the proposed method. The proposed training beam design and channel estimation method can be applied both to the initial channel learning and to efficient channel tracking after the channel is initially learned by some other method.

\section{REFERENCES}

[1] V. Venkateswaran and A. van der Veen "Analog beamforming in MIMO communications with phase shift networks and online channel estimation," IEEE Trans. Signal Process., vol. 58, no. 8, pp. 4131 - 4143, 2010.

[2] O. E. Ayach, R. W. Heath Jr., S. Abu-Surra, S. Rajagopal, and Z. Pi, "Low complexity precoding for large millimeter wave MIMO systems," in Proc. IEEE Int. Conf. Commun., Jun. 2012.

[3] A. Alkhateeb, O. E. Ayach, G. Leus, and R. W. Heath, "Hybrid precoding for millimeter wave cellular systems with partial channel knowledge," in Proc. Inf. Theory and Appl. Workshop., (San Diego, CA), 2013.

[4] A. Alkhateeb, O. E. Ayach, G. Leus, and R. W. Heath, "Channel Estimation and Hybrid Precoding for Millimeter Wave Cellular Systems," submitted to IEEE J.Sel. Topics Signal Process., arXiv preprint arXiv:1401.7426,2014.

[5] C. Doan, S. Emami, D. Sobel, A. Noknejad and R. Brodersen, "Design considerations for 60 GHz CMOS radios," IEEE Commun. Mag., vol. 42, no. 12, pp. 132 - 140, 2004.

[6] I. E. Telatar, "Capacity of multi-antenna Gaussian channels," European Trans. Telecommun., vol. 10, no. 6, pp. 585 - 595, 1999.

[7] B. Hassibi and B. M. Hochwald, "How much training is needed in multiple-antenna wireless links?," IEEE Trans. Inf. Theory, vol. 49, pp. 951-963, Apr. 2003.

[8] J. H. Kotecha and A. M. Sayeed, “Transmit signal design for optimal estimation of correlated MIMO channels," IEEE Trans. Signal Process., vol. 52, pp. 546 - 557, Feb. 2004.

[9] J. Choi, D. J. Love, and P. Bidigare, "Downlink training techniques for FDD massive MIMO systems: Open-loop and closed-loop training with memory," IEEE J. Sel. Topics Signal Process., vol. 8, pp. 802 - 814, Oct. 2014. 
[10] S. Noh, M. Zoltowski, Y. Sung and D. J. Love, "Pilot beam pattern design for channel estimation in massive MIMO systems,” IEEE J. Sel. Topics Signal Process., vol. 8, pp. 787 - 801, Oct. 2014.

[11] J. So, D. Kim, Y. Lee, and Y. Sung, "Pilot signal design for massive MIMO systems: A received signal-to-noise-ratio-based approach,” accepted to IEEE Signal Process. Lett., 2014.

[12] W. U. Bajwa, J. Haupt, A. M. Sayeed, and R. Nowak, "Compressed channel sensing: a new approach to estimating sparse multipath channels," Proc. IEEE., vol. 98, pp. 1058 - 1076, Jun. 2010.

[13] G. Tauböck and F. Hlawatsch, "Compressed sensing based estimation of doubly selective channels using a sparsityoptimized basis expansion," in Proc. Eur. Signal Process. Conf., (Switzerland), Aug. 2008.

[14] J. Wang, Z. Lan, C. Pyo, T. Baykas, C. Sum, M. Rahman, J. Gao, R. Funada, F. Kojima, H. Harada and Shuzo Kato, "Beam codebook based beamforming protocol for multi-Gbps millimeter-wave WPAN systems," IEEE Trans. J. Sel. Areas Commun., vol. 27, no. 8, pp. 1390 - 1399, 2009.

[15] S. Hur, T. Kim, D. Love, J. Krogmeier, T. Thomas and A. Ghosh, "Millimeter wave beamforming for wireless backhaul and access in small cell networks," IEEE Trans. Commun., vol. 61, no. 10, pp. 4391 - 4403, 2013.

[16] L. Tong, B. Sadler, and M. Dong, "Pilot-assisted wireless transmissions: General model, design criteria, and signal processing,” IEEE Signal Processing Mag., vol. 21, no. 6, pp. 12 - 25, 2004.

[17] M. L. Puterman, Markov decision processes: Discrete stochastic dynamic programming. John Wiley \& Sons, 1994.

[18] J. Seo, Y. Sung, G. Lee, and D. Kim, "Pilot beam sequence design for channel estimation in millimeter-wave MIMO systems: A POMDP framework," submitted to ICC 2015, Sep. 2014. Available at http://arxiv.org/abs/1409.8434.

[19] A. M. Sayeed, "Deconstructing multiantenna fading channels," IEEE Trans. Signal Process., vol. 50, pp. 2563 - 2579, Oct. 2002.

[20] Akbar M. Sayeed and Vasanthan Raghavan, "Maximizing MIMO capacity in sparse multipath with reconfigurable antenna arrays,” IEEE J. Sel. Topics Signal Process., vol. 1, pp. 156 - 166, Jun. 2007.

[21] H. V. Poor, “An Introduction to Signal Detection and Estimation,” New York: Springer, 1994.

[22] Y. Sung, L. Tong, and H. V. Poor, "Neyman-Pearson detection of Gauss-Markov signals in noise: Closed-form error exponent and properties," IEEE Trans. Inf. Theory, vol. 52, pp. 1354 - 1365, Apr. 2006.

[23] R. D. Smallwood and E. J. Sondik, "The optimal control of partially observable Markov processes over a finite horizon," in Operations Research, vol. 21, pp. 1071-1088, 1973.

[24] E. Monahan, "State of the ArtA Survey of Partially Observable Markov Decision Processes: Theory, Models, and Algorithms," Management Science. , vol. 28, no. 1, pp. 1 - 16, 1982.

[25] S. M. Ross, Applied probability models with optimization applications. Courier Dover Publications, 1970.

[26] A. Cassandra, M. L. Littman and N. L. Zhang, "Incremental pruning: A simple, fast, exact method for partially observable Markov decision processes," Proc. Thirteenth Ann. Conf. on Uncertainty in Artificial Intelligence., pp. 54 - 61, Morgan Kaufmann Publishers Inc., 1997.

[27] J. Pineua, G. Gordon, and S. Thrun, "Point-based value iteration: An anytime algorithm for POMDPs," in Proc. Int. Joint Conf. Artificial Intelligence, vol. 3, pp. 1025 - 1032, 2003.

[28] H. Kurniawati and D.Hsu and W. Lee, "SARSOP: Efficient Point-Based POMDP Planning by Approximating Optimally Reachable Belief Spaces,” in Proc. Robot. Sci. Syst, 2008.

[29] T. Smith and R. Simmons, "Point-based POMDP algorithms: Improved analysis and implementation," arXiv preprint arXiv:1207.1412, 2012. 